\title{
Extracting Nonlinear Dynamics from Psychological and Behavioral Time Series Through HAVOK Analysis
}

\author{
Robert G. Moulder, Elena Martynova, and Steven M. Boker \\ University of Virginia
}

\begin{abstract}
Analytical methods derived from nonlinear dynamical systems, complexity, and chaos theories offer researchers a framework for in-depth analysis of time series data. However, relatively few studies involving time series data obtained from psychological and behavioral research employ such methods. This paucity of application is due to a lack of general analysis frameworks for modeling time series data with strong nonlinear components. In this article, we describe the potential of Hankel alternative view of Koopman (HAVOK) analysis for solving this issue. HAVOK analysis is a unified framework for nonlinear dynamical systems analysis of time series data. By utilizing HAVOK analysis, researchers may model nonlinear time series data in a linear framework while simultaneously reconstructing attractor manifolds and obtaining a secondary time series representing the amount of nonlinear forcing occurring in a system at any given time. We begin by showing the mathematical underpinnings of HAVOK analysis and then show example applications of HAVOK analysis for modeling time series data derived from real psychological and behavioral studies.
\end{abstract}

Keywords: HAVOK, chaos theory, nonlinear dynamics, time series, attractors

Broadly speaking, the primary goal of psychological and behavioral science is understanding and defining the nature of human experience. However, achieving this goal is generally difficult as humans are inherently complex systems. Although biological differences between individual humans may be relatively small compared to biological differences between humans and other animals, the set of all emotions, behaviors, and cognitive processes that define individual human experiences at any given time is immense. Within different individuals, these defining factors are also sensitive to external influences. One person may be highly extraverted, enjoy extreme sports, and experience chronic depression symptoms, while another person may be highly introverted, enjoy cooking at home, and be in prime mental health, even if these individuals are monozygotic twins (Asbury, Dunn, Pike, \& Plomin, 2003; Turkheimer \& Waldron, 2000). To further add to this complication, not only do these defining factors of human experience vary from person to person, but these factors also vary within a single individual across time and context. This should not be surprising as humans do not live static lives in controlled environments. To the contrary, humans change over time, altering their behavior in response

Robert G. Moulder, Department of Psychology, University of Virginia; Elena Martynova, Department of Psychology, University of Virginia; Steven M. Boker, Department of Psychology, University of Virginia; NOTE: This paper has not been peer reviewed. Please do not copy or cite without author's permission. to both external and internal influences. Each of these influences compound with one another in ways that affect individual humans both temporarily and permanently, at both short and long time scales. These compounding influences interact with one another to create multiple nonlinear relationships between all variables that define how an individual is experiencing life at any given moment. Therefore, studying only the linear components of human experience at single occasions is not sufficient to give a fully accurate account of the dynamics of human nature. Human experience is complex, nonlinear, and dynamic and must be studied as such.

Complex nonlinear dynamical systems contain intrinsic relationships that are not easily understood by linear processes. As one aspect changes, other aspects may increase, decrease, increase and then decrease, oscillate, or show other complicated trends. Due to these nonlinear relationships, complex nonlinear dynamical systems may show unique properties that would be impossible to observe in linear systems. These properties include multiple points of equilibrium, bifurcations, sensitive dependence on initial conditions, and many others (e.g., Danvers, Wundrack, \& Mehl, 2020; Kan, Ploeger, Raijmakers, Dolan, \& Van Der Maas, 2010). Arguably, each of these properties map to common aspects of behavior and show up in anecdotal expressions in casual conversation, such as individuals reporting feelings of instability, having "ups and downs", or experiencing high degrees of uncertainty (Barton, 1994; Boker, 2013; Vallacher \& Nowak, 1997).

The long term steady-state behavior of a dynamical sys- 
tem is defined as that system's equilibrium. Dynamical systems with multiple equilibrium have multiple possible long term steady-state behaviors. While linear dynamical systems tend to evolve towards a single low energy or "comfortable" state as time goes on, nonlinear dynamical systems may have multiple possible "comfortable" points. Systems with multiple equilibria have more than one possible long term state and perturbations to that system may switch the system from one state to another. In human systems, this may refer to individuals switching between psychological states such as focused versus unfocused, psychophysiological states such as relaxed vs. stressed, or behavioral states such as cooperative versus independent task solving.

Bifurcations occur when these equilibrium points increase in number or change qualities as a variable changes. Consider an example of such behavior in human systems. Individuals' depression levels after a traumatic event may either regulate back to levels of pre-traumatic event levels, or may change to be in a new long-term depressive state. Sensitive dependence on initial conditions occurs when the equilibria of a complex dynamical system becomes so complex that even small deviations in that system lead to large differences over time. In human systems this can be seen with the lifespan trajectories of many individuals with similar childhood experiences having quite different adult lives. While such complex equilibrium dynamics appear to coincide with numerous human behaviors, none of these dynamics may be accurately modeled with linear analyses. Consequently common analysis techniques across psychological and behavioral science, such as linear regression, may often fail to be able to capture essential complex and nonlinear properties of human systems.

Researchers have recognised this need to study the psychological and behavioral aspects of human experience as complex nonlinear dynamical systems and have begun collecting intensive time series data on individual participants. While behavioral time series data has been historically difficult to collect, modern wearable data collection devices, video recording equipment, and smart devices such as smartphones offer researchers more flexibility of study design and an ease of data collection not previously possible in regard to time varying phenomena displayed by humans. For instance, Gruebler and Suzuki (2014) designed minimally intrusive wearable device for the detection of positive facial expression. This device, worn on the side of an individual's head much like a set of headphones, and accompanying algorithm allows for researchers to collect on the fly emotion data with reliability in the range of human coders (Kappa $=.95$ ). Ramseyer and Tschacher (2011) developed a freely available software tool, motion energy analysis (MEA), for quantifying the amount of motion in video recordings of participants. The MEA software tool utilizes pixel differencing methods to quantify motion in selected regions of a video stream. MEA has been used in a number of behavioral studies with topics ranging from patients and care-provider interactions in psychotherapy sessions and medical settings, to team-based cooperative problem solving (e.g., Behrens et al., 2019; Hamel et al., 2018; Nelson, Grahe, Ramseyer, \& Serier, 2014). Recently, Daniel et al. (2020) used ecological momentary assessment (EMA) methodologies to model the effect of cognitive bias modification strategies on the behaviors of individuals high in social anxiety. To achieve this goal, Daniel et al. (2020) used smartphones to collect daily questionnaire data for each participant. Such innovative data collection techniques as those previously discussed are becoming increasingly more accessible to researchers interested in studying human dynamics, allowing for a noted rise in publications utilizing time series data derived from human studies ${ }^{1}$. These new techniques have driven researchers to broaden the scope of questions asked about human systems over time, driving a need for data analysis techniques suitable for modeling time series data derived from human studies.

Classical time series analysis techniques based on autoregressive (AR), moving average (MA), autoregressive integrated moving average (ARIMA) or autoregressive conditionally heteroscedastic $(\mathrm{ARCH})$ frameworks have been previously used by researchers to study humans over time. These families of models are discrete time methods for analysing time series data. That is, these methods relate a data point at a specific time to data points from a previous time based on some model structure in the form:

$$
x_{t}=f\left(x_{t-1}\right)
$$

Discrete time methods build a temporal model by breaking down time series data into components of stability (moving average components), relatedness to past states of the data (autoregressive components), cyclical components (seasonality), conditional components (cross-regression), and others depending on the method used by a researcher. Researchers using such methods have had success in modeling various aspects of human behavior over time. Feldman, MagoriCohen, Galili, Singer, and Louzoun (2011) used ARIMA modeling techniques to model the synchronization between the hearts of 3-month old infants and their mothers during a free play exercise. Engert et al. (2011) used cross-correlation analysis to determine the response time course of different biological markers of stress in human saliva (cortisol and alpha-amylase) after participants engaged in the Trier Social Stress test and found that these biological markers take more than 10 minutes to reach peak correlation values. Other researchers have used vectorized versions of these analyses

\footnotetext{
${ }^{1}$ A Google Scholar search for both the terms "time series" and "psychology" from 1990 - 1999 yields 14,400 results, from 2000 - 2009 yields 56,900 results, and from 2010 - 2019 yields 67,100 results.
} 
to model large systems of variables across multiple individuals simultaneously to study clinically relevant markers of psychological disorders such as depression and problematic eating behaviors (Epskamp, Waldorp, Mõttus, \& Borsboom, 2018; Wild et al., 2010).

Although valuable tools for understanding how qualities of human experience change over time, discrete time models have a number of limitations that significantly impact both the reliability of such models, as well as the kinds of questions that researchers may ask when using such models. For instance, de Haan-Rietdijk, Voelkle, Keijsers, and Hamaker (2017) found that the results of discrete time models are sensitive to sampling interval. That is, the differences in time elapsed between successive points of data used to fit a discrete time model will change the values of parameters within a discrete time model. Other researchers such as Weiss (1984) have shown that sampling interval and sampling rate of a given phenomenon can also influence the results of such models. Issues such as these mean that for many discrete time models, unless all time points are measured at equal intervals and sampling rate is kept constant, interpretation of parameters across different studies is difficult. Such constrains may limit the generalizability of findings derived from discrete time models. To counter this, some researchers opt to use a continuous time approach instead, derived from dynamical systems theory Boker, Neale, and Rausch (2004); Driver and Voelkle (2018); van Montfort, Oud, and Voelkle (2018).

\section{Dynamical Systems Modeling in Psychological and Be- havioral Research}

Continuous time models in psychological and behavioral research tend to rely on classical dynamical systems theory to describe time-varying psychological phenomena (see van Montfort et al., 2018). In this framework, psychological and behavioral processes are modeled as ordinary differential equations of the form:

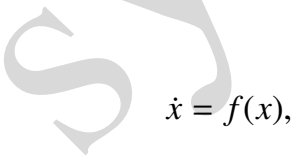

where $x$ is the derivative of variable $x$ with respect to time and $f(x)$ is some function of $x$ defining the rate of change of $x$. Continuous time models overcome the limitations of discrete time models through the use of explicit specification of $f(x)$ and that the outcome variable, $\dot{x}$, may be considered "instantaneous" change as opposed to change at a future time point. That is, in continuous time modeling the expected change in $x$ at any time point is a function of $x$ at that same time point. This framework may be extended to include multiple variables through vectorization as:

$$
\begin{aligned}
& \dot{x_{1}}=f\left(x_{1}, x_{2}, \ldots, x_{k}\right) \\
& \dot{x_{2}}=f\left(x_{1}, x_{2}, \ldots, x_{k}\right) \\
& \dot{\mathbf{x}}=f(\mathbf{x}) \quad=\quad x_{2}=f\left(x_{1}, x_{2}, \ldots, x_{k}\right), \\
& \dot{x_{k}}=f\left(x_{1}, x_{2}, \ldots, x_{k}\right)
\end{aligned}
$$

where $\dot{\mathbf{x}}$ is a vectorized form of $\dot{x_{1}} \ldots \dot{x_{k}}$ and $f(\mathbf{x})$ is a vectorized form of $f\left(x_{1}, x_{2}, \ldots, x_{k}\right)$ for $k$ variables. In general, there are two classes of ordinary differential equations that may be used to describe time varying phenomena: linear ordinary differential equations (defining linear dynamical systems) and nonlinear ordinary differential equations (defining nonlinear dynamical systems).

Linear dynamical systems in psychological and behavioral research. Linear dynamical systems are a versatile class of models for understanding temporal characteristics such as exponential growth/decay and regulation behaviors. Linear dynamical systems may be expressed as linear differential equation models with $\mathbf{f}(\mathbf{x})$ being a linear combination of $\mathbf{x}$ such that $\dot{\mathbf{x}}=\mathbf{x A}$, where $\mathbf{A}$ is a matrix of coefficients. Although relatively simplistic, linear differential equation models have been used to model a variety of physical phenomena across the sciences, from the motion of springs and pendula to the growth rates of bacteria. In the social and behavioral sciences, such models can account for a wide variety of time series behaviors that may interest substantive researchers. The use of dynamical systems models also offers researchers the ability to apply models of physical phenomena to psychological processes that have common anecdotal relations to the physical world, such as "feeling in a rut" or "unstable".

For instance, researchers have used linear dynamical systems models to understand qualities of regulation in psychological and behavioral data that may be difficult to assess in a discrete time framework. Steele and Ferrer (2011) used second-order latent differential equation models to understand emotional coregulation behaviors of romantic partners as paired springs, finding that individuals in romantic relationships regulate their own positive and negative affect, as well as the emotional states of their partners. Using a similar method, Hu, Boker, Neale, and Klump (2014) found that the hormone estradiol is associated with regulation of emotional eating behaviors, and that this relationship is moderated by individuals' negative affect. The daily negative affect and stress ratings of older individuals has been demonstrated by Deboeck and Bergeman (2013) to behave as first-order linear differential equations with stochastic error terms, traditionally used to model the quantity of liquid in a reservoir. These applications of dynamical systems to psychological research demonstrates the versatility of a dynamical systems view of psychological and behavioral processes and gives researchers the ability to relate psychological and behavioral processes to phenomena observed in physical systems. 


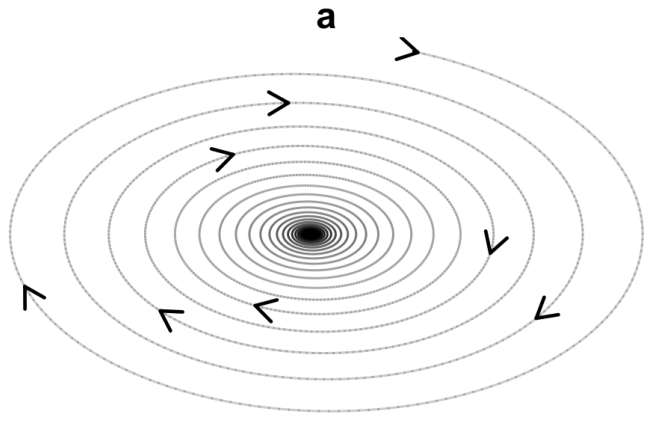

C

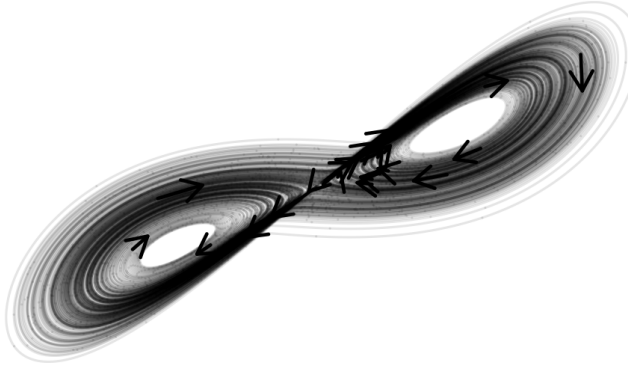

e

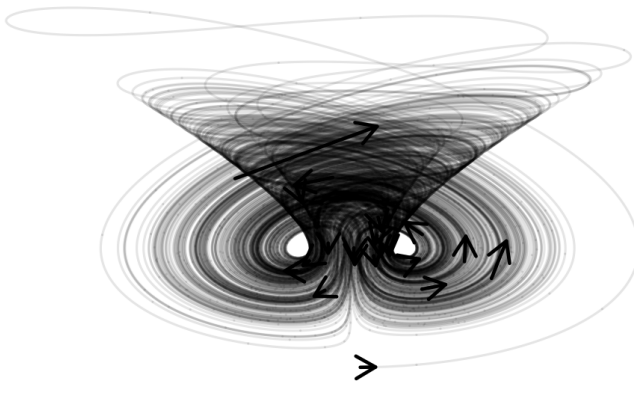

b
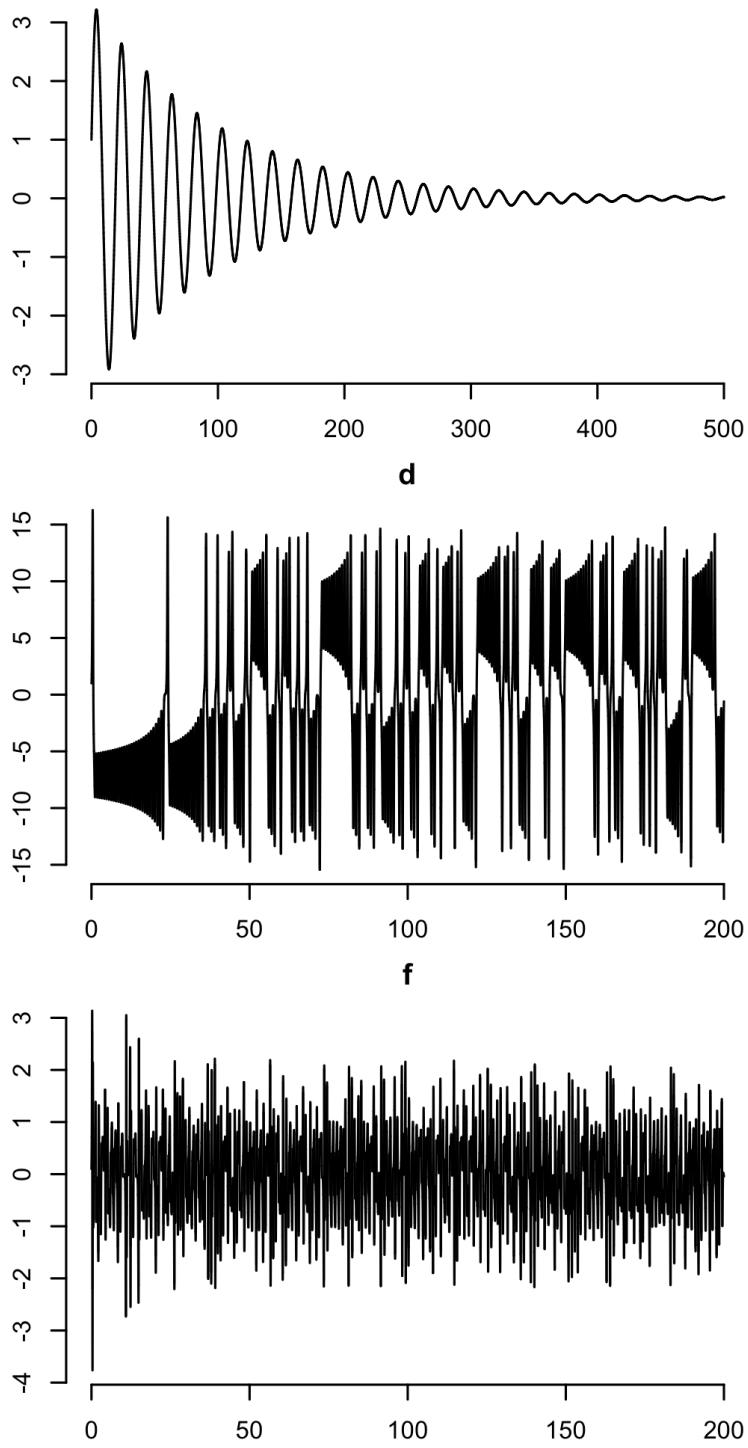

Figure 1. Example attractors (left column) with associated time series (right column). Attractor (a) represents an attractor for a linear system with associated time series (b). Attractors (c) and (e) represents attractors for two nonlinear systems with associated time series (d) and (f). Arrows represent the direction of a trajectory in phase-space. Time series of nonlinear attractors are able to display more complicated behaviors than time series from systems with linear attractors.

Nonlinear dynamical systems in psychological and behavioral research. Although quite versatile, linear dynamical systems models defined by sets of linear differential equations are generally not able to model highly complex phenomena. This is because the attractors of complex phenomena do not in general tend to be singular points in phasespace (Figure $1 \mathrm{~A}$ ), but are more complicated structures, Figure $1 \mathrm{~B} \& \mathrm{C}$. The phase-space of a dynamical system is a set of coordinates defining behavior of the dynamical system at any given time. An attractor is a point, set of points, or structures in phase-space for which all points move toward in the stable case, or away from in the unstable case, as time goes on. The attractors of complex nonlinear dynamical systems are manifolds that can display highly complex and difficult to describe geometries. A consequence of this is that nonlinear dynamical systems may not show such simplistic behaviors as growth or regulation as time goes on, but may show a wide variety of more complex and theoretically interesting behaviors, known collectively as nonlinearities (Vallacher \& Nowak, 1997).

While the time series of a nonlinear dynamical system may show erratic behavior across time, the attractors of these 
systems may be highly structured. As all of the time based dynamics of a nonlinear dynamical system are contained within that system's attractor, inferential methods modeling the topology of attractors in phase-space may be more intuitive and possibly more informative than inferential methods modeling time series behaviors solely across time. Due to this, attractor reconstruction from observed data is at the heart of many methods for understanding complex nonlinear dynamical systems (Abraham, 1997; Broomhead \& King, 1986; Takens, 1981). Once the attractor manifolds of nonlinear dynamical systems reach a certain level of complexity, such as the Lorenz attractor, these systems are considered to be chaotic (Liu, Liu, Liu, \& Liu, 2004; Lorenz, 1963).

The study of nonlinear behaviors and nonlinearities in time series have been beneficial to understanding many physical, psychological, and behavioral systems. Indeed, such methods are necessary to study systems that show behaviors more complex than simple growth or regulation. For example, Chow, Witkiewitz, Grasman, and Maisto (2015) showed that a cusp catastrophe model (a form of bifurcation in multiple dimensions) could be applied to psychological and behavioral systems such as attitude change and problematic alcohol consumption. In an attempt to model complexity within dyadic conversation, Ashenfelter, Boker M., Waddell, and Vitanov (2009) used multi-fractal analysis to disentangle processes of human head-motion patterns at different time scales. Though relatively limited in number, studies such as these demonstrate the potential theoretical scope and impact of studying nonlinearities within human systems.

However, a major limitation of nonlinear dynamical systems analysis is that in many cases these models cannot be expressed in the form $\dot{\mathbf{x}}=\mathbf{x A}$. This limits the ability of researchers to model nonlinear dynamical systems in a regression framework as would be possible with linear dynamical systems. The lack of general methodology for modeling nonlinear dynamical systems in psychological and behavioral research has lead to relatively few studies employing nonlinear dynamical systems theory to answer questions involving human systems, even though the potential gain in explanatory power of yarious psychological processes by the use of nonlinear dynamical systems modeling is immense (Barton, 1994).

Additionally, the results of nonlinear dynamical systems models are highly dependent on the functional form of $\mathbf{f}(\mathbf{x})$, as even subtle changes in $\mathbf{f}(\mathbf{x})$ may lead to dramatically different attractor structures in phase-space. Thus, researcher choice of a $\mathbf{f}(\mathbf{x})$ that best models a specific phenomenon may heavily influence inferences derived from the study of the phenomenon. If a researcher chooses a proper $\mathbf{f}(\mathbf{x})$ to model a phenomenon, most methods of studying nonlinear differential equations focus only on the analysis of local aspects of dynamical systems as opposed to global aspects of these systems. That is, most methods of studying nonlinear dynamical systems isolate specific regions of a system and study the dynamics close to that region (local), as opposed to studying the behavior of a system as a whole (global). Local aspects of a dynamical system are generally studied through the use of Taylor series expansion about fixed-points in phase-space (Nagarajan, 2005). It is generally more difficult to linearize an entire phase-space in such a way that preserves all global dynamics (e.g., Day, Junge, \& Mischaikow, 2004; Hsu, 1980). These qualities limit the widespread use of nonlinear dynamical systems theory to the whole of psychological and behavioral science, and restrict the ability of psychological researchers to develop hypotheses regarding nonlinearities of their particular systems of interest. An analysis framework is needed for modeling and recovering nonlinearities within psychological and behavioral time series that does not depend on a priori specification of $\mathbf{f}(\mathbf{x})$, reliably performs attractor reconstruction, and holistically models a system in its entirety, as opposed to only local aspects.

\section{Recovery of Nonlinear and Chaotic Dynamics using Lin- ear Models: HAVOK Analysis}

Developments by Brunton, Brunton, Proctor, Kaiser, and Kutz (2017) have applied nonlinear dynamical systems theory to recover nonlinearities from time series data. Their method, known as the Hankel alternative view of Koopman (HAVOK) analysis, is a general method for equation free modeling of dynamical systems by decomposing any set of measurements of a dynamical system into linear components and a forcing term such that even highly nonlinear systems with measurement error may be modeled in the form:

$$
\dot{\mathbf{x}}=\mathbf{x A}+\mathbf{u}(\mathbf{t}) \mathbf{B},
$$

where $\mathbf{u}(\mathbf{t})$ is a forcing term that perturbs $\mathbf{x}$ at time $t, \mathbf{A}$ is a square matrix of parameters mapping $\mathbf{x}$ to $\dot{\mathbf{x}}$, and $\mathbf{B}$ is a row vector of parameters for $\mathbf{u}(\mathbf{t})$. HAVOK analysis decomposes a time series such that $\mathbf{u}(\mathbf{t})$ contains all the time series' nonlinear information. Thus, nonlinearities such as bifurcations, regime switching, and bursting in a dynamical system at any given time may be inferred by the relative magnitude of $\mathbf{u}(\mathbf{t})$. We believe that HAVOK analysis shows promise as being an indispensable tool for psychological and behavioral researchers due to its generality and relative ease of application.

The remainder of this article seeks to solidify this claim and is structured as follows: First, we define and discuss the mathematical underpinnings of HAVOK analysis, including attractor reconstruction and Koopman operator theory. We then show an example of HAVOK analysis on simulated data from a theoretical mathematical system (the Lorenz attractor), data derived from an electroencephalogram study, data derived from human head motion, and a time series of selfreported depression symptoms. Finally, we end with current limitations of HAVOK analysis, future directions for further 
refinement of HAVOK analysis for psychological and behavioral science, and speculation on applications of HAVOK analysis for understanding modern psychological research issues.

\section{HAVOK Analysis}

HAVOK analysis may be broken down into the following steps:

i) Preliminary attractor reconstruction;

ii) Attractor decomposition into eigen time series;

iii) Separation of linear and nonlinear components;

iv) Linear state-space representation of nonlinear dynamics.

The first step in HAVOK analysis is to reconstruct the attractor of a given dynamical system. Attractor reconstruction is a method of reconstructing the phase-space dynamics of a dynamical system given at least one quality of that system is sufficiently measured across time. Though there are many ways of performing attractor reconstruction, most rely on the seminal works of Whitney (1936) and Takens (1981). Takens' theorem of attractor reconstruction states that, given a single well-chosen variable of a dynamical system measured over time, an attractor may be reconstructed such that the reconstructed attractor is diffeomorphic to the true attractor of the dynamical system. That is, a manifold may be reconstructed from a single 1-dimensional time series that shares all topological properties of the true underlying attractor of that dynamical system, even if the dynamical system is of dimensionality much greater than 1. Takens' theorem accomplishes this by embedding a univariate time series $x$ into a matrix $\phi(x)$ of a dimension greater than 1 . This so-called embedding dimension defines the number of columns of $\phi(x)$ and will result in a good approximation of the underlying attractor of a system given that the embedding dimension is of sufficient size and a researcher chooses an appropriate time lag. This process of reconstructing a structure from measurements is similar to that of latent differential equation modeling popular in psychological science (Boker et al., 2004; $\mathrm{Hu}$ et al., 2014). Since its inception, Takens' theorem has been expanded to include multivariate systems as well as systems with large stochastic components and is a cornerstone of modern dynamical systems research (e.g., Mezić \& Banaszuk, 2004).

HAVOK analysis leverages Takens' theorem by constructing an embedding matrix, $\phi(x)=\mathbf{H}$, such that $\mathbf{H}$ is a Hankel matrix. That is, each row-wise element of $\mathbf{H}$ is lagged to the previous row by 1 unit of time and each column-wise element of $\mathbf{H}$ is lagged to the previous element by 1 unit of time. For example, consider a set of measurements $x_{t}$ from a dynamical system such that $x_{t} \in[1,2,3,4,5,6,7,8,9]$. One possible Hankel Matrix construction for $x_{t}$ is then:

$$
\mathbf{H}=\left[\begin{array}{llllll}
1 & 2 & 3 & 4 & 5 & 6 \\
2 & 3 & 4 & 5 & 6 & 7 \\
3 & 4 & 5 & 6 & 7 & 8 \\
4 & 5 & 6 & 7 & 8 & 9
\end{array}\right] .
$$

Under Takens' theorem, a matrix of this form may be sufficient to reconstruct the attractor of $x$ if the proper time lag happens to be 1 sample. Under the HAVOK analysis framework this time lag value is always 1 sample, significantly reducing the amount of researcher involvement needed in constructing H (Takens, 1981).

While valuable for understanding the dynamics of the dynamical system underlying $x$, the phase-space defined by $\mathbf{H}$ still represents a nonlinear measurement space, and thus inferences from linear analysis techniques are problematic. However, HAVOK analysis creates a linear space that closely approximates $\mathbf{H}$ though the use of a low-rank approximation to the Koopman operator, $\mathcal{K}$. This process decomposes $\mathbf{H}$ into a set of representative time series of eigen time delay coordinates through singular value decomposition (SVD). The linear space defined by the SVD of $\mathbf{H}$ is then considered for further analyses (Bollt, Li, Dietrich, \& Kevrekidis, 2018; Broomhead \& King, 1986; Brunton et al., 2017; Brunton, Brunton, Proctor, \& Kutz, 2016; Koopman, 1931).

\section{Decomposition into Eigen Time Series}

Koopman operator theory is a complementary view of dynamical systems to the traditional geometric view of attractor reconstruction common in dynamical systems analysis. Koopman operator theory, and the associated Koopman operator $\mathcal{K}$, are a means of converting a nonlinear measurement space into a linear measurement space (Koopman, 1931). Specifically, Koopman operator theory offers the potential of representing any finite dimensional nonlinear dynamical system as an infinite dimensional linear system by mapping the entire Hilbert space of the nonlinear system to an infinite dimensional linear space. Through the lens of Koopman operator theory, nonlinear dynamical systems embedded in high dimensions may be modeled as the time-based dynamics of observable values derived from these spaces (Brunton et al., 2016; Budišić, Mohr, \& Mezić, 2012). That is, each observed value of a dynamical system at a given time, $x_{t}$, may be seen as the result of applying a nonlinear measurement function $g(\cdot)$ to the true underlying dynamical system. The Koopman operator $\mathcal{K}$, is a theoretical infinite dimensional linear function that advances $g\left(x_{t}\right)$ to $g\left(x_{t+1}\right)$ in the discrete case (or defines the derivative of $g\left(x_{t}\right)$ in the continuous case), such that $\mathcal{K} g\left(x_{t}\right)=g\left(x_{t+1}\right)$. In this way, $\mathcal{K}$ is a linear map of nonlinear measurement function $g\left(x_{t}\right)$ to $g\left(x_{t+1}\right)$. This effectively infinitely expands and flattens a nonlinear measurement space 


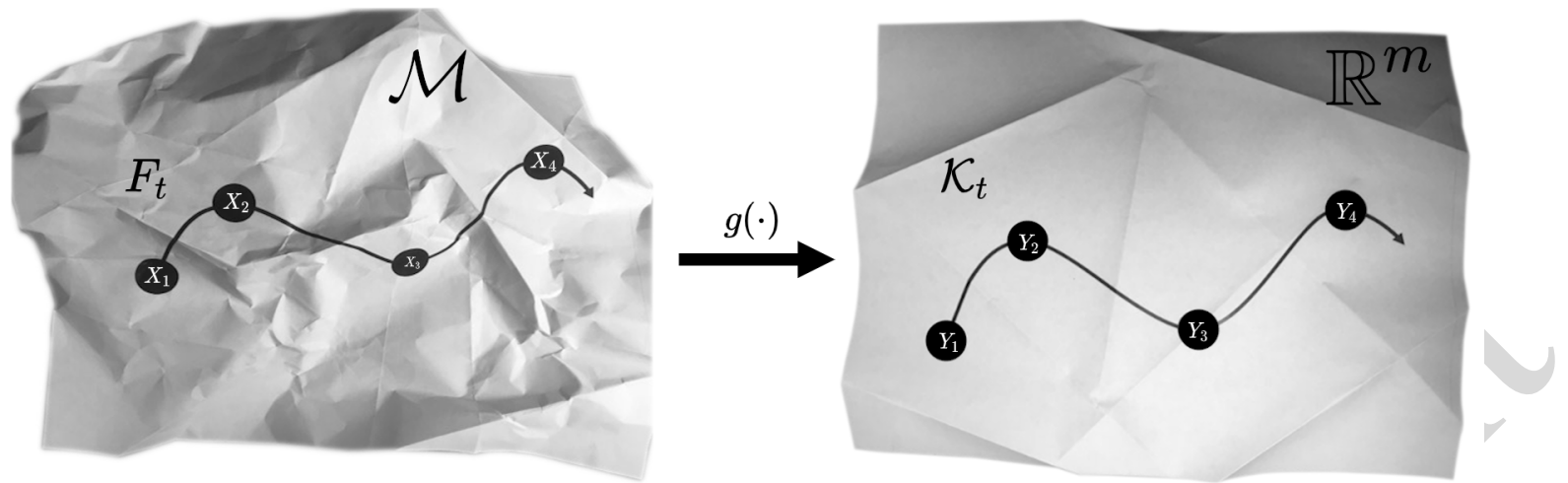

Figure 2. Koopman operator theory represented as a function progressing across a crumpled sheet of paper. The crumpled paper on the left represents a high (possibly infinite) nonlinear measurement space, $\mathcal{M}$, with each ridge on the sheet of paper acting as a dimension of $\mathcal{M}$. The function $F_{t}$ maps successive observations, $X_{i}$ to one another in $\mathcal{M}$. The function $g(\cdot)$ imparts a smooth flattening on $F_{t}$ in $\mathcal{M}$, yielding a lower, finite dimensional space $\mathbb{R}^{m}$. This process is similar to flattening out a crumpled sheet of paper. Not all ridges will be removed by flattening, but the influence of the ridges on the flattened paper on what is printed on the paper is minimal. The Koopman operator $\mathcal{K}_{t}$ then maps successive observations of $g\left(X_{i}\right)=Y_{i}$ to one another in $\mathbb{R}^{m}$.

until the system in that space also behaves in a linear fashion, Figure 2.

A primary step in HAVOK analysis is the construction of the Hankel matrix $\mathbf{H}$. $\mathbf{H}$ is a matrix with $K$ rows and $(N-K+1)$ columns, composed of $N$ measurements, $x_{t}$ : $t \in[1,2,3, \ldots, N]$, of a dynamical system that defines a nonlinear measurement space. Brunton et al. (2017) showed that successive values of $\mathbf{H}$ may be viewed as $\mathcal{K}$ successively operating on the value of $\mathbf{H}_{1,1}$ as follows:

$$
\begin{aligned}
\mathbf{H} & =\left[\begin{array}{cccc}
x_{1} & x_{2} & \ldots & x_{N-K+1} \\
x_{2} & x_{3} & \ldots & x_{N-K+2} \\
x_{3} & x_{4} & \ldots & x_{N-K+3} \\
\vdots & \vdots & \ddots & \vdots \\
x_{K} & x_{K+1} & \ldots & x_{N}
\end{array}\right] \\
& =\left[\begin{array}{ccccc}
x_{1} & \mathcal{K} x_{1} & \mathcal{K}^{2} x_{1} & \ldots & \mathcal{K}^{N-K} x_{1} \\
\mathcal{K} x_{1} & \mathcal{K}^{2} x_{1} & \mathcal{K}^{3} x_{1} & \ldots & \mathcal{K}^{N-K+1} x_{1} \\
\mathcal{K}^{2} x_{1} & \mathcal{K}^{3} x_{1} & \mathcal{K}^{4} x_{1} & \ldots & \mathcal{K}^{N-K+2} x_{1} \\
\vdots & \vdots & \vdots & \ddots & \vdots \\
\mathcal{K}^{K-1} x_{1} & \mathcal{K}^{K} x_{1} & \mathcal{K}^{K+1} x_{1} & \ldots & \mathcal{K}^{N-1} x_{1}
\end{array}\right] .
\end{aligned}
$$

Singular value decomposition may then be applied to $\mathbf{H}$ to achieve a finite dimensional linear space that is a good approximation to the infinite dimensional linear space mapped through the use of $\mathcal{K}$ :

$$
\mathbf{H}=\mathbf{U} \boldsymbol{\Sigma} \mathbf{V}^{\prime} .
$$

That is, the SVD of $\mathbf{H}$ creates a finite linear space containing a highly accurate approximation to the infinite linear space created through the use of $\mathcal{K}$. This Koopman invariant subspace is defined by the columns of the eigen time series in the left $(\mathbf{U})$ and right $(\mathbf{V})$ decomposition matrices. These eigen time series are ordered in $\mathbf{U}$ and $\mathbf{V}$ by their contribution to the variance explained in $\mathbf{H}$ and may be considered latent variables in line with those extracted by principal components analysis (Wall, Rechtsteiner, \& Rocha, 2003). Matrix V may then be used to reconstruct an attractor with topologically consistent properties to the true attractor of a dynamical system by plotting the leading columns in $\mathbf{V}$ in some orthogonal space (Brunton et al., 2017) ${ }^{2}$.

\section{Obtaining Intermittent Forcing Values}

After obtaining the eigen time series of $\mathbf{H}$ contained in $\mathbf{U}$ and $\mathbf{V}$, the next step in the HAVOK analysis of a time series is to reduce the rank of the columns of $\mathbf{V}$ so as to optimize reconstruction of the Koopman invariant space conditional on the number of columns in $\mathbf{V}$. This is necessary since, if $\mathbf{H}$ has noise components, these noise components will quickly begin to overshadow the true variation extracted from the columns of the $\mathbf{U}$ and $\mathbf{V}$ matrices obtained by SVD. There are a number of methods designed specifically for optimizing the number of retained columns of $\mathbf{V}$ (e.g., Gavish \& Donoho, 2014; Horn, 1965). We denote the number of columns of $\mathbf{V}$ retained from this processes as $r$ and the associated reduced $\mathbf{V}$ matrix as $\mathbf{V}_{\mathbf{r}}$. Derivatives of $\mathbf{V}_{\mathbf{r}}, \dot{\mathbf{V}}_{\mathbf{r}}$, may then be estimated through methods such as $\mathrm{n}^{\text {th }}$-order finite difference or generalized local linear approximations (Boker, Deboeck, Edler, \& Keel, 2010; Mattsson \& Nordström, 2004).

\footnotetext{
${ }^{2}$ Other methods of decomposition are also valid, including frequency domain decompositions such as dynamic mode decomposition (DMD). In this case the $\mathbf{U}$ matrix will be on the frequency domain.
} 
At this point, a linear system with parameter matrix $\mathbf{A}$ can be approximated through ordinary least squares:

$$
\dot{\mathbf{V}}_{\mathbf{r}}=\mathbf{V}_{\mathbf{r}} \mathbf{A}
$$

where $\mathbf{V}_{\mathbf{r}}^{\prime}$ is the transpose of $\mathbf{V}_{\mathbf{r}}$ and $\mathbf{A}=\left(\mathbf{V}_{\mathbf{r}}^{\prime} \mathbf{V}_{\mathbf{r}}\right)^{-1} \mathbf{V}_{\mathbf{r}}^{\prime} \dot{\mathbf{V}}_{\mathbf{r}}$. However, if HAVOK analysis is performed correctly then this model will show a sub-optimal fit due primarily to the rightmost column of $\mathbf{V}_{\mathbf{r}}, \mathbf{v}_{\mathbf{r}}$, and its associated parameters in $\mathbf{A}, \mathbf{a}_{\mathbf{r}}$. If instead, these values are removed from $\mathbf{V}_{\mathbf{r}}$ and $\mathbf{A}$, and used to construct a forcing term $\mathbf{u}(\mathbf{t})$ :

$$
\mathbf{u}(\mathbf{t}) \mathbf{B}=\mathbf{v}_{\mathbf{r}} \mathbf{a}_{\mathbf{r}}{ }^{\prime}
$$

this forcing component may then be added to the remaining components of equation 8 to create the final HAVOK model:

$$
\dot{\mathbf{V}}(\mathbf{t})=\mathbf{V}(\mathbf{t}) \mathbf{A}+\mathbf{u}(\mathbf{t}) \mathbf{B}
$$

where $\dot{\mathbf{V}}(\mathbf{t})$ is the first $r-1$ columns of $\dot{\mathbf{V}}_{\mathbf{r}}$ and $\mathbf{V}(\mathbf{t})$ is the first $r-1$ columns of $\mathbf{V}_{\mathbf{r}}$. In general, $\mathbf{u}(\mathbf{t})$ will show a spiking behavior. The magnitude of these spikes denote the amount of active forcing $\mathbf{u}(\mathbf{t})$ is applying to $\mathbf{V}(\mathbf{t}) \mathbf{A}$, and is thus indicative of when $x_{t}$ is demonstrating nonlinear behaviors. Equation 10 may be viewed as a linear regression model defining the time based dynamics of $\mathbf{V}(\mathbf{t})$ on $\dot{\mathbf{V}}(\mathbf{t})$ conditional on $\mathbf{v}_{\mathbf{r}} \mathbf{a}_{\mathbf{r}}{ }^{\prime}$. This same model may also be created in a state-space modeling framework (Chow, Ho, Hamaker, \& Dolan, 2010; Hunter, 2018). The response to equation 10 given a set of initial conditions equal to the first row of $\dot{\mathbf{V}}(\mathbf{t})$ may then be used assess the relative fit of the HAVOK model implied time series. This fit can be assessed against both the original time series $x_{t}$ and the first eigen time series of $\mathbf{v}_{\mathbf{r}}$ with metrics such as $R^{2}$.

The intuition behind the process of creating the HAVOK regression model in equation 10 may be understood as a set of counterfactual information that separates signal from noise, and then separates linear signal components from nonlinear signal components. The SVD solution obtained by decomposing $\mathbf{H}$ into a set of representative eigen time series is important as these eigen time series are sorted by the amount of variance explained in $\mathbf{H}$, with potentially more noisy components being pushed to further right columns of $\mathbf{V}$. Of note is that SVD guarantees $\mathbf{V}$ is ordered by linear explanatory power to $\mathbf{H}$. Selection of $\mathbf{V}_{\mathbf{r}}$ from $\mathbf{V}$ retains columns of $\mathbf{V}$ that best explain $\mathbf{H}$ with minimal influence of noise. However, because $\mathbf{H}$ has nonlinear components as well as linear components, these nonlinear components are also forced more right in $\mathbf{V}$ by SVD, but explain more variance in $\mathbf{H}$ than noise (Bhattacharya \& Kanjilal, 1999). Thus, when equation 9 is fit, if time series data $x_{t}$ has reliably nonlinear components then the final column of $\mathbf{V}_{\mathbf{r}}$ will not reliably be linearly related to $\dot{\mathbf{V}}_{\mathbf{r}}$ due to nonlinearities present in $x_{t}$. By instead using these nonlinearities as a forcing term, $\mathbf{u}(\mathbf{t})$, HAVOK is able to use information of the nonlinearities in $x_{t}$ to perturb $\mathbf{V}(\mathbf{t}) \mathbf{A}$ such that equation 10 is able to model nonlinear systems with high degrees of precision while using only linear equations. The forcing term $\mathbf{u}(\mathbf{t})$ is then of interest to psychological and behavioral researchers as $\mathbf{u}(\mathbf{t})$ contains all necessary information of the occurrence and magnitude of nonlinear behaviors in $x_{t}$.

\section{HAVOK Analysis Results}

HAVOK analysis is an analytical framework allowing researchers the opportunity to gain insights into nonlinear behaviors of time series data. Researchers may use HAVOK analysis for singular time series analysis, or apply HAVOK to multiple time series for comparative purposes. By conducting a HAVOK analysis of time series derived from psychological and behavioral systems researchers gain the ability to reconstruct attractors for these systems, estimate a forcing term representing the amount of nonlinear forcing in a system at any given time, and represent any system (linear or nonlinear) as a linear model.

Attractor reconstruction offers researchers a topological representation of their systems. Once an attractor has been reconstructed for a time series, researchers may engage in both qualitative and quantitative analysis of the attractor's form. For qualitative analysis of attractors, researchers may describe structures on the attractor and the relation of these structures to psychological and behavioral phenomena. For instance, the attractor of neuron models tend to show a tightly clustered region (representing no spiking) and a long-tailed or spiral structures indicative of spiking (e.g., Hindmarsh \& Rose, 1984; Hodgkin \& Huxley, 1952). For quantitative analysis of attractors, researchers may use analytic methods such as recurrence analysis, maximal Lyapunov exponent estimation, or measures of attractor similarity to obtain values quantifying aspects both within and between attractors (e.g., Brick, Gray, \& Staples, 2018; Lefèvre, Lepresle, \& Chariot, 2014; Peppoloni, Lawrence, Ruffaldi, \& Valero-Cuevas, 2017; Timofejeva, Poskuviene, Cao, \& Ragulskis, 2018).

Unlike most other methods for attractor reconstruction, attractors reconstructed through HAVOK analysis do not require specialized methods for embedding a time series into a high dimensional space since the embedding matrix for HAVOK analysis is always a Hankel matrix. This quality of HAVOK analysis greatly reduces the effort required by researchers in order to obtain stable and valid attractor reconstructions from time series data. That is, HAVOK analysis makes valid attractor reconstruction from time series more standardized across experiments since there are far fewer choices a researcher must make to reconstruct an attractor while using HAVOK analysis compared to other methods of attractor reconstruction (Lu, Hunt, \& Ott, 2018; Takens, 1981).

Forcing term estimation offers researchers a secondary 


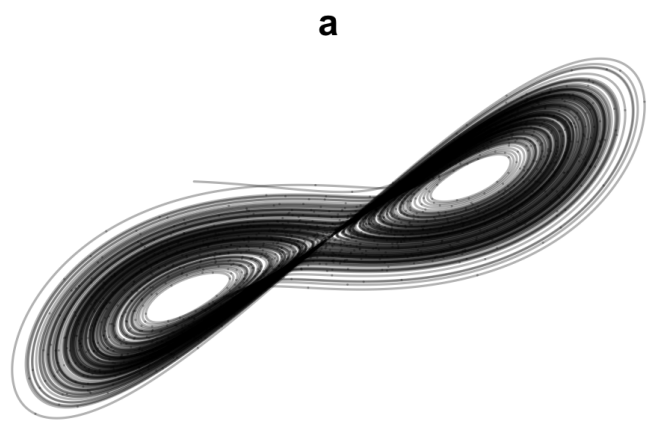

C

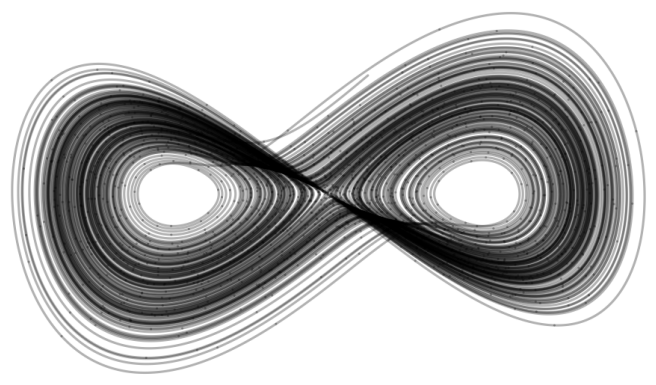

e

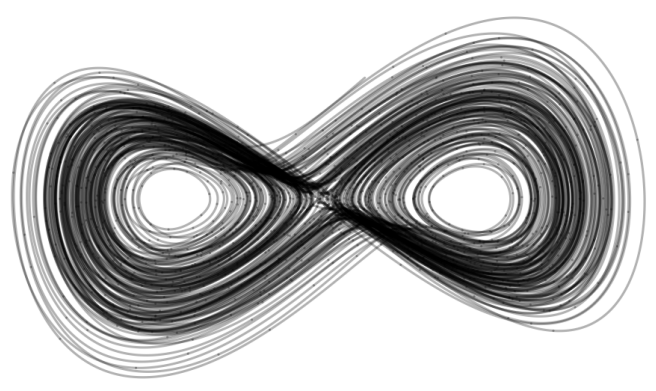

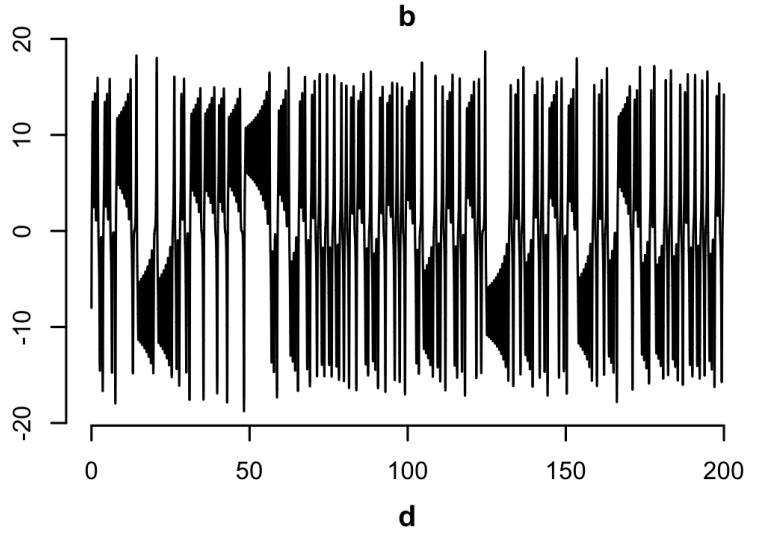
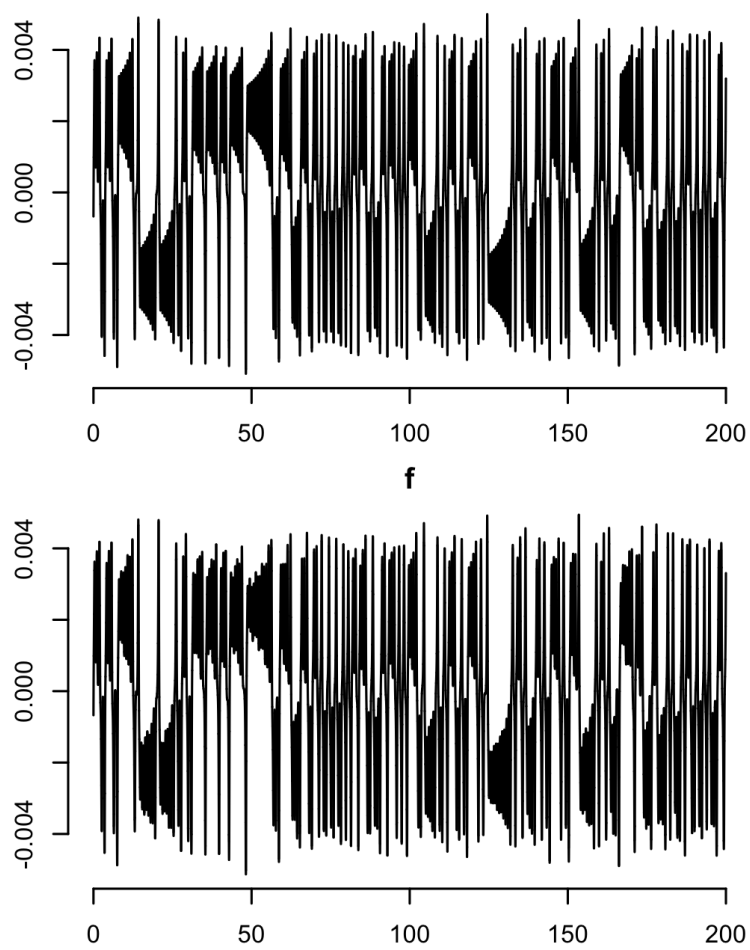

Figure 3. Creation of a HAVOK analysis of a Lorenz system. Attractors for each phase of HAVOK are in the left column and associated time series are on the right. Attractor (a) represents the true underlying attractor formed by knowledge of the $X$, $Y$, and $Z$ coordinates of the Lorenz system. Time series (b) is the associated $X$ time series of this system used during analysis. Attractor (c) is the reconstruction of (a) given only (b) obtained from the SVD of $\mathbf{H}$. Time series (d) is the first column of $\mathbf{V}$. Attractor (e) is the attractor reconstructed from the HAVOK regression model. Time series (f) is the output of the HAVOK regression model.

time series representing the amount of nonlinear forcing occurring in a system at any given time. Coupled with attractor reconstruction, this forcing term may be used to define regions of an attractor/time series that represent nonlinearities. Identification of nonlinear regions allows psychological or behavioral phenomena to be related to nonlinearities and general complexity; qualities many researchers consider highly understudied. (Barton, 1994; Guastello \& Liebovitch, 2009; Richardson, Dale, \& Marsh, 2014). Additionally, ex- ternal variables may be used to model or be modeled by a forcing term. If an external variable is influenced by a forcing term (i.e., the forcing term is a dependent variable) then that variable may be sensitive to nonlinearities within a given system under study. If an external variable influences the forcing term (i.e., the forcing term is an independent variable) then that external variable may be understood as influencing nonlinear behavior in a system and may possibly be used as a control parameter. Such control parameters may be 
of interest to behavioral modification such as just-in-time interventions (Nahum-Shani et al., 2018; Vallacher \& Nowak, 1997).

Finally, the theoretical ability of HAVOK analysis to represent any nonlinear time series as a linear model is a simple method for constructing a mathematical representation of any dynamical system; decomposing a system into linear components, $\mathbf{V}(\mathbf{t})$, and a vector representing nonlinear forcing, $\mathbf{u}(\mathbf{t})$. This linear representation allows for dynamical systems analysis of nonlinear systems in an equation free format. That is, unlike many common dynamical systems analysis methods, HAVOK analysis does not require researchers to know a specific set of differential equations that may have generated an observed time series under study. It is also of note that many time series are not entirely linear or entirely nonlinear. Under the HAVOK analysis framework, linear qualities may be studied through further analysis of $\mathbf{V}(\mathbf{t})$ and the associated $\mathbf{A}$ parameter matrix, while nonlinear qualities may be studied through further analysis of $\mathbf{u}(\mathbf{t})$ and the associated $\mathbf{B}$ parameter matrix.

To demonstrate the general applicability and information gained through the use of HAVOK analysis to studying nonlinearities in psychological and behavioral data, we next present analysis steps and results of HAVOK analysis applied to the Lorenz system, a well known chaotic dynamical system (Lorenz, 1963). This analysis will represent a ground truth test as the nonlinearities of the Lorenz system are well known and visually apparent in the time series. We then show HAVOK analysis applied to three data sets from psychological research: an EEG signal derived from a study employing a go/no-go task, a dyadic data set derived from human head motion, and a data set derived from self-reported feelings of self-doubt from an $N=1$ study of depression symptomology during a reduction in medication. The application of HAVOK analysis to these data sets will show how HAVOK analysis may be used to obtain meaningful insights for psychological and behavioral researchers. For each data set, HAVOK analysis will be used to reconstruct an attractor and obtain a nonlinear forcing term. Both of these qualities will be inspected for meaningful structures. All analyses were performed with $\mathrm{R}$ with the havok package available at: https://github.com/RobertGM111/havok. Additionally, all code required to replicate all analyses in this article is openly available at: https://osf.io/hzvfq/.

\section{Lorenz System Analysis}

The Lorenz system is a set of 3 coupled first-order nonlinear differential equations and is one of the first mathematical systems used to study chaotic dynamics (Lorenz, 1963). The set of equations defining the Lorenz system may be written as:

$$
\begin{aligned}
\dot{X} & =\sigma(Y-X), \\
\dot{Y} & =X(\rho-Z)-Y, \\
\dot{Z} & =X Y-\beta Z,
\end{aligned}
$$

where $X, Y$, and $Z$ are position states in 3D space, and $\sigma$, $\rho$, and $\beta$ are constants. The Lorenz system displays chaotic behavior for a number of values of $\sigma, \rho$, and $\beta$. For this example we chose $\sigma=10,=28$, and $b=\frac{8}{3}$ in line with Brunton et al. (2017). In total, 200,000 data points were simulated from this system. The Lorenz system has a number of benefits as a toy model of chaos. A specific benefit for this example is that the major nonlinear component in the Lorenz system is visually apparent in both the time series and in the reconstructed attractor (i.e., lobe switching in phasespace and upward/downward shifts in the time series of the Lorenz systems), Figure 3 (a) and (b). If HAVOK analysis can indeed recover nonlinear dynamics in a time series, then HAVOK analysis should be able to recover when this lobe switching behavior occurs.

HAVOK analysis was applied to the $X$ time series derived from this Lorenz system. Similar to Brunton et al. (2017), an $r$ of 15 was chosen and $\mathbf{H}$ was created with 100 rows and 199,901 columns. An attractor reconstruction from $\mathbf{V}_{\mathbf{r}}$ is presented in Figure 2 (b), and the associated first eigen time series of this system derived from $\mathbf{V}_{\mathbf{r}}$ is presented in Figure 2 (c). The attractor reconstruction successfully retains all topological qualities as the true underlying attractor of this system, even though the HAVOK analysis was unaware of the true underlying attractor and was only utilizing a single time series, $X$, as opposed to all three time series comprising the Lorenz system. The squared Pearson correlation $\left(R^{2}\right)$ of the first eigen time series of $\mathbf{V}_{\mathbf{r}}$ and $X$ is .93, indicating an excellent fit. Additionally, the columns of the $\mathbf{U}$ matrix (known as U-modes) are presented in Figure 4. These columns represent a set of basis functions for the rates of change of the rows of the $\mathbf{H}$. An optimal HAVOK model will ideally show similar smooth polynomial functions defining this basis (Brunton et al., 2017).

The bottom section of Figure 5 represents the absolute value of forcing term, $\mathbf{u}(\mathbf{t})$, derived from HAVOK analysis. The absolute value of $\mathbf{u}(\mathbf{t})$ represents the magnitude of nonlinear forcing occurring in $X$ and spikes in the absolute value of $\mathbf{u}(\mathbf{t})$ preceed lobe switching in $X$ (i.e., the major nonlinear event of the Lorenz system). Once $\mathbf{u}(\mathbf{t})$ reaches a threshold, this lobe switching event occurs in $X$. In this way, forcing may be conceptualized as being "ON" or "OFF" if $\mathbf{u}(\mathbf{t})$ is above/below a predetermined cutoff value. We find that in general a cutoff value between $\pm S D[\mathbf{u}(\mathbf{t})]$ and $\pm 2 S D[\mathbf{u}(\mathbf{t})]$ gives a good representation of when forcing values are "ON" versus "OFF". Post estimation, researchers may use $\mathbf{u}(\mathbf{t})$ as a dependent or independent variable in further analyses to determine the influences $\mathbf{u}(\mathbf{t})$ on other variables and influenc- 


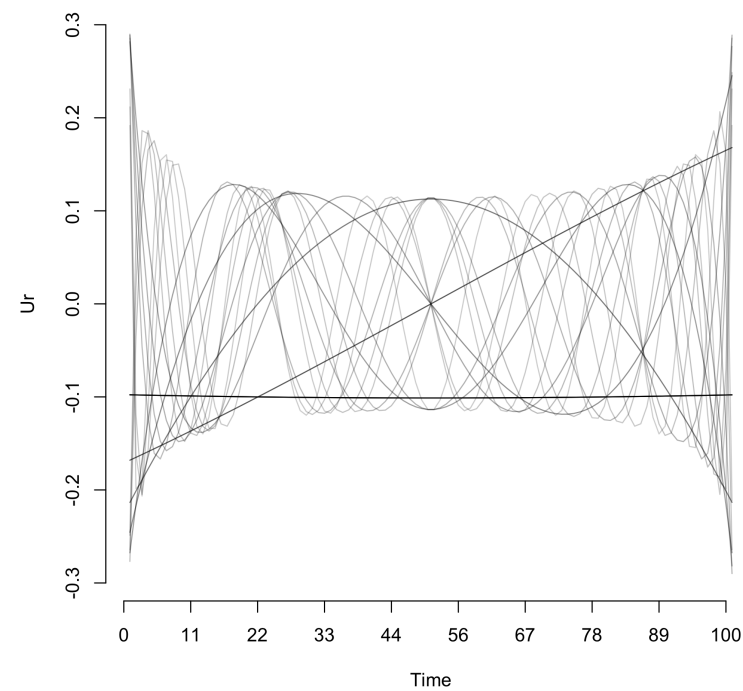

Figure 4. U-modes of the Lorenz system studied in this article. Each mode represents a polynomial function defining the basis of the rate of change of the $\mathbf{H}$ associated with this HAVOK analysis.

ing factors of other variables on $\mathbf{u}(\mathbf{t})$. Interestingly, because spikes in $\mathbf{u}(\mathbf{t})$ occur slightly before nonlinearities in the observed time series, other variables may be used to predict when a nonlinearity will occur before a nonlinearity is observed in $X$. The resulting HAVOK regression model seen in Figure 3 (e) and (d) shows a strong fit to both the original time series $\left(R^{2}=.93\right)$ and the first eigen time series of $\mathbf{V}_{\mathbf{r}}$ $\left(R^{2}=.99\right)$.

\section{EEG Analysis}

Data from real systems do not behave as ideal mathematical systems with known nonlinearities, such as the Lorenz system. Real data sets show varying degrees of noise and regularity. Researchers also generally do not have a closed form solution to the underlying process of a given data set. EEG is a common data collection technique that is known to have some levels of noise, relatively high regularity, and show chaotic dynamics with a known nonlinearity presented as spiking behavior (Kunhimangalam, Joseph, \& Sujith, 2008). Brunton et al. (2017) has previously shown that HAVOK analysis is able to model and recover the nonlinear behavior of EEG signals. We replicate this finding here in a separate EEG data set obtained from the OpenNeuro project available at: https://openneuro.org/datasets/ds002680/versions/1.0.0.

We utilized data collected by Delorme, Rousselet, Macé, and Fabre-Thorpe (2004) from a study of EEG activity during a go/no-go task. Data sampled at $1000 \mathrm{~Hz}$ from the FP region of a single participant was first passed through a highpass filter of $.1 \mathrm{~Hz}$ and then a low-pass filter of $40 \mathrm{~Hz}$ to achieve a stable waveform. These data were then additionally down-sampled to $400 \mathrm{~Hz}$ for the purposes of this example. We then conducted a HAVOK analysis on this data set. We chose an $r$ of 4 and $\mathbf{H}$ was created with 10 rows and 39,991 columns.

The reconstructed attractor and first eigen time series of this data set are presented in Figure 6 (a) and (b). The results of the HAVOK regression model are displayed in Figure 5 (c) and (d). The reconstructed attractor obtained through HAVOK analysis of this EEG data is similar to that obtained from other studies modeling EEG signals (Stam, 2005). The topology of this attractor, similar in form to a "monkey's fist" knot, shows two interesting structures: a tight ball representing brain activity between spikes, and large arcs from this ball representing spiking behavior. The first eigen time series of this data correlates well with the original input EEG time series $\left(R^{2}=.94\right)$, and the time series derived from the HAVOK regression model fits both the eigen time series and the original signal well, $R^{2}=.96$ and $R^{2}=.94$, respectively. Figure 7 displays a section of the obtained forcing term for this EEG time series. As with the Lorenz system, the forcing term for this EEG time series shows a spiking behavior immediately preceding the known nonlinear events in the EEG time series. This analysis demonstrates the potential for HAVOK analysis to reconstruct attractors and define nonlinear regions of time series derived from psychophysiological studies.

\section{Head Motion During Dyadic Conversation Analysis}

We have shown in the Lorenz and EEG cases that HAVOK analysis recovers meaningful insight about the dynamics of these systems, replicating similar to insights obtained from these systems in previous studies. Here, we present a novel application of HAVOK analysis for understanding interpersonal dynamics and nonverbal interaction. We conducted a HAVOK analysis of two time series derived from angular velocities of head motion obtained from two participants engaged in dyadic conversation with one another. Each time series was sampled at $83 \mathrm{~Hz}$ for 5 -minutes and standardized into z-scores. We chose an $r$ of 3 for each time series and $\mathbf{H}$ for each time series was created with 5 rows and 16,397 columns.

We applied HAVOK analysis to each participant's data separately. For participant 1, the first eigen time series of this data correlates well with the original input motion time series $\left(R^{2}=.98\right)$, and the time series derived from the HAVOK regression model fits both the eigen time series and the original motion time series well, $R^{2}=.98$ and $R^{2}=.97$ respectively. For participant 2 , the first eigen time series of this data also correlates well with the original input motion time series $\left(R^{2}=.99\right)$, and the time series derived from the HAVOK regression model fits both the eigen time series and the original motion time series well, $R^{2}=.91$ and $R^{2}=.91$ 

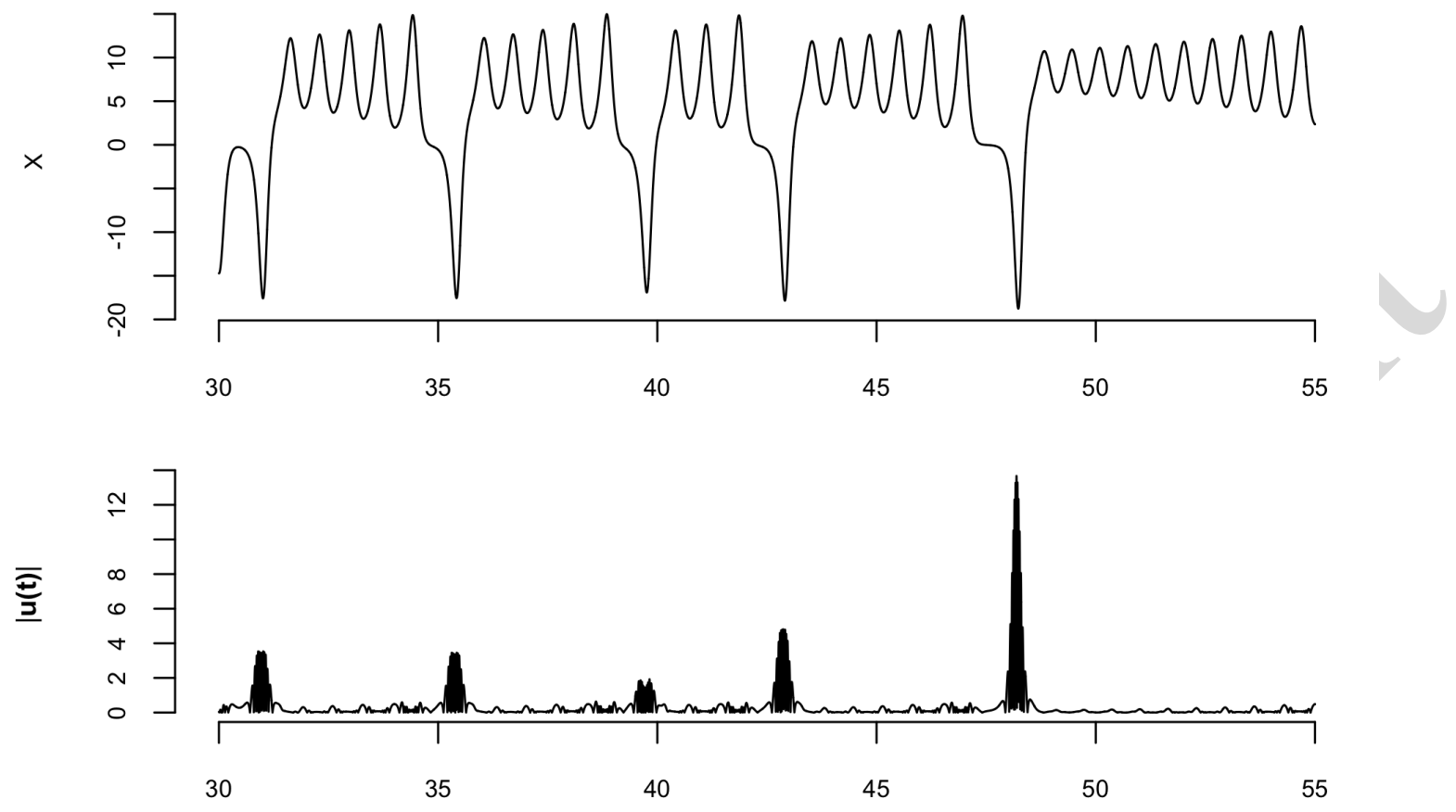

Time

Figure 5. Plots of a section of the $X$ variable of a Lorenz system (top) with the associated forcing term $\mathbf{u}(\mathbf{t})$ derived from HAVOK analysis of $X$. Spikes in the forcing term denote when this Lorenz system becomes highly nonlinear and precede the lobe switching behavior indicative of the Lorenz system.

respectively. Attractor reconstructions for each participant are shown in Figure 8 (a) and (c) for participant 1 and 2 respectively, along with the associated motion time series.

The attractors for each participant show a similar shape, indicating that there may be a general high dimensional topological structure representing how humans move their heads during conversation, or at least that this specific conversation has such a structure. Each participant's attractor shows a spiraling pattern around a centroid, with tendrils moving along either side. These tendril structures are associated with large forcing values (i.e., are nonlinearities) and possibly represent short head nodding behavior and speech (centroid behavior), and large motions possibly indicative of laughter or surprise (tendrils). Additionally, these attractors mirror one another in phase-space, which may be indicative of conversation symmetry (Ashenfelter et al., 2009; Boker \& Rotondo, 2002). It does not seem like a stretch of the imagination to consider both attractors defining each participant's conversation dynamics to be parts of a larger attractor defining the dynamics of the dyad in conversation. To our knowledge, this is the first evidence of symmetrical attractor structures forming during conversation. Further analyses of these topologies may yield further insight into nonverbal dynamics during conversation.

When studying the forcing terms of each participant, seen in Figure 9, it appears that most often when one participant's forcing term is active (i.e., greater than $S D[\mid \mathbf{u}(\mathbf{t}||])$, that the other participant's forcing term is inactive (i.e., less than $S D[|\mathbf{u}(\mathbf{t})|])$. This indicates that a single individual at any given time carries the majority of the nonlinear information in motion dynamics during a conversation. These active nonlinear phases may be due to a participant leading the conversation and the clear separation between linear and nonlinear regions between participants may be indicative of turn-taking behaviors, however more research is needed to confirm the validity of this hypothesis. Additionally, there are short segments in which both participants have active forcing terms. These segments may indicate a number of behaviors such as joint acknowledgement, laughing together, or other forms of mutual motion dynamics. Further analysis may find that these peaks in the forcing terms of each individual may vary by conversation topic, participant familiarity, or type of relationship (e.g., romantic, mentor/protege, or friendship).

\section{Self-Doubt Analysis}

As a final exemplar analysis, we present a HAVOK analysis of a psychologically relevant, but technically difficult to model data set of 1476 self-reported self-doubt assessments across 8 months. In this $N=1$ study conducted by Wich- 

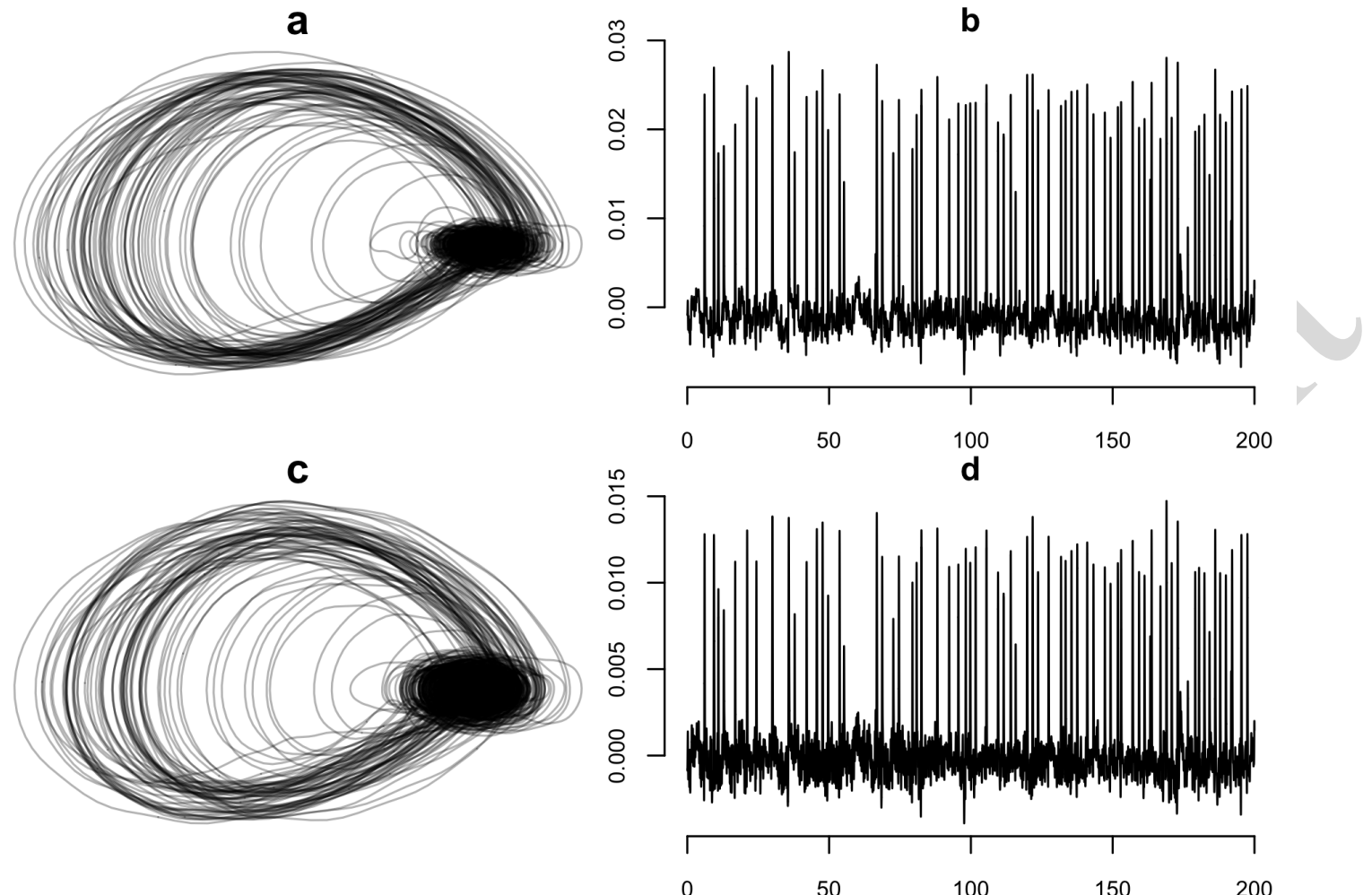

Figure 6. A HAVOK analysis of EEG data. Attractor (a) is an attractor reconstruction from (b) given only time series data obtained from the SVD of $\mathbf{H}$. Time series (b) is the first column of $\mathbf{V}$. Attractor (c) is the attractor reconstructed from the HAVOK regression model. Time series (d) is the output of the HAVOK regression model.

ers and Groot (2016), a single participant was asked to rate the truthfulness of the statement "I feel indecisive." measured on a scale of 1 (Not) to 7 (Very) over a period in which the participant had their medication reduced (Kossakowski, Groot, Haslbeck, Borsboom, \& Wichers, 2017; Wichers \& Groot, 2016). Data similar to these would not be uncommon in intensive longitudinal measurement of individuals or in long-term ecological momentary assessment studies. Intensively measured survey data are known to be both highly nonlinear and/or full of noise or measurement artifacts and thus would provide a meaningful challenge to the HAVOK analysis framework (Santangelo, Bohus, \& Ebner-Priemer, 2014; Wichers \& Groot, 2016).

We applied HAVOK analysis to this data set twice, once with an $r$ of 2 and an $\mathbf{H}$ with 2 rows and 1471 columns (model A), and once with a $r$ of 2 and an $\mathbf{H}$ with 10 rows and 1463 columns (model B), Figure 10. In model A, the first eigen time series from the HAVOK analysis shows a strong fit to the original time series $\left(R^{2}=.69\right)$, and the time series derived from the HAVOK regression model shows a perfect fit to the data $\left(R^{2}=1\right)$. This indicates a lack of degrees of freedom presented by model $\mathrm{A}$, even though there is strong fit. As such, we ded with a less well fitting model, model B, that preserved the overall behavior of the original time series but, given the larger number of rows in $\mathbf{H}$, gave a result similar to a smoothed version of this time series. The first eigen time series from model B shows a relatively weak fit to the original time series $\left(R^{2}=.20\right)$. The time series derived from the HAVOK regression model shows a strong fit to the first eigen time series $\left(R^{2}=.90\right)$ and a relatively weak fit to the original time series $\left(R^{2}=.11\right)$. We attribute this relatively poor fit to the discrete nature of the data along with added noise.

The attractor derived from the $\mathbf{V}_{\mathbf{r}}$ matrix of this system does not show strong structural regularities, however there appears to be a region in which trajectories appear to cluster. Values outside of this region are associated with active nonlinear forcing. However, nonlinear forcing is active throughout most of the time series indicating that this system is generally nonlinear. This highly active forcing term may be expected given $r=2$ (i.e., only two columns of $\mathbf{V}$ for this system were retained after SVD). In the case of $r=2$ for equation 10, both $\mathbf{A}$ and $\mathbf{B}$ are scalars. Thus the relative values of $\mathbf{V}(\mathbf{t}) \mathbf{A}$ to $\mathbf{u}(\mathbf{t}) \mathbf{B}$ will define how linear/nonlinear the system behaves. More precise and frequent measurements of this system would result in a larger $r$. 

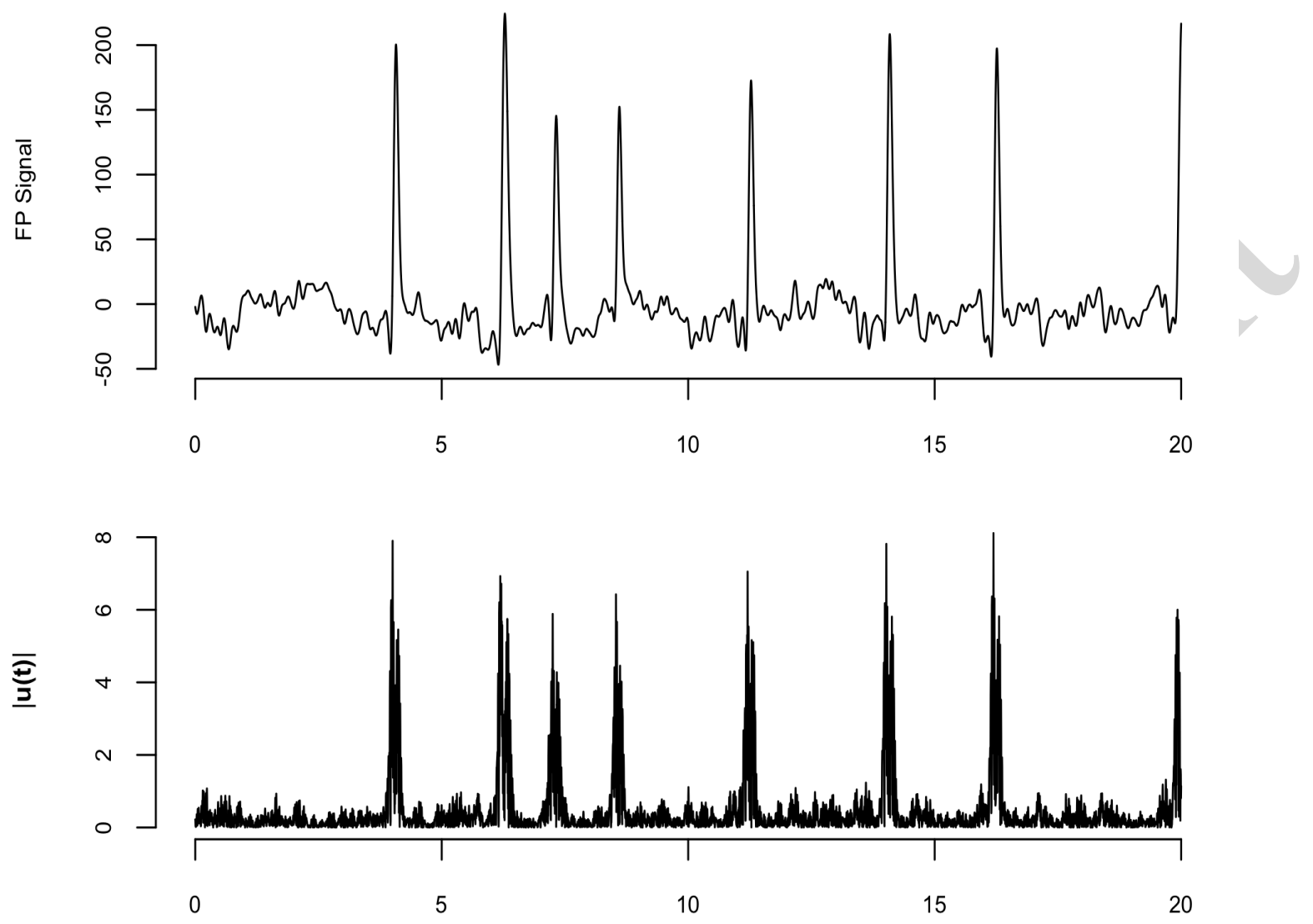

Time

Figure 7. Plots of a section of an EEG signal (top) with the associated forcing term $\mathbf{u}(\mathbf{t})$ derived from HAVOK analysis of this signal. Spikes in the forcing term denote when this EEG signal becomes highly nonlinear and precedes spiking behavior in the EEG signal.

\section{Discussion}

Many aspects of human behavior complex, nonlinear, and change over time. However, the study of this change is difficult, partially due to a lack of a cohesive and general modeling framework for complex nonlinear dynamical systems. We have suggested that HAVOK analysis, a method derived in statistical mechanics, engineering, and control theory, as a possible modeling framework to ease this difficulty. Researchers seeking to study nonlinearities within their systems of interest are able to utilize HAVOK to reliably decompose time series data into linear components and a forcing term that contains all of the nonlinear information of the system. We have also created an $\mathrm{R}$ package (currently housed on GitHub) to further the usability of HAVOK analysis on psychological and behavioral data.

HAVOK analysis requires selecting 2 hyper-parameters: the number of rows in $\mathbf{H}$ and $r$. The choice of the number of rows in $\mathbf{H}$ will affect the smoothness of the resulting eigen time series from a HAVOK analysis, with larger values inducing more smoothing. The choice of $r$ denotes how many columns of $\mathbf{V}$ will be retained for analysis, with the $r$-th column of $\mathbf{V}$ being reserved to construct the forcing term $\mathbf{u}(\mathbf{t})$. If $r$ is too small the HAVOK analysis will not properly separate the nonlinear components of a time series. TIf $r$ is too large the columns of $\mathbf{V}$ associated with noise will be retained and modeled as $\mathbf{u}(\mathbf{t})$. While a reasonable $r$ may be automatically optimized during model construction, the proper selection of the number of rows in $\mathbf{H}$ must first be specified by a researcher. In our experience, these two parameters do not seem to have a relationship suitable for convex optimization. Thus, researchers seeking to apply current implementations of HAVOK analysis should try a number of models while looking specifically to: (a) maximize the relationship of the first eigen time series of $\mathbf{V}$ with their time series of interest by choosing an appropriate number of rows for $\mathbf{H}$, (b) attempt multiple $r$ values and assess the relationship of 
a

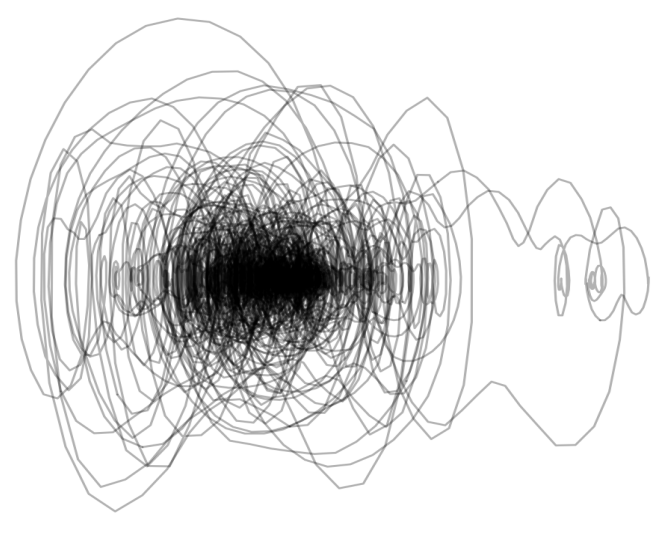

C

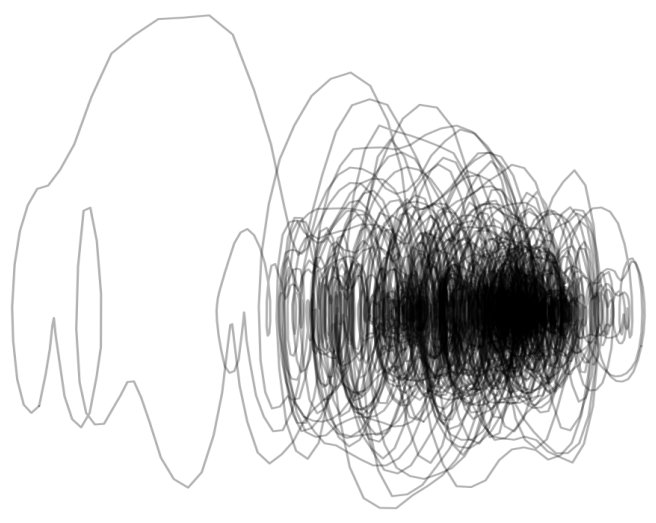

b
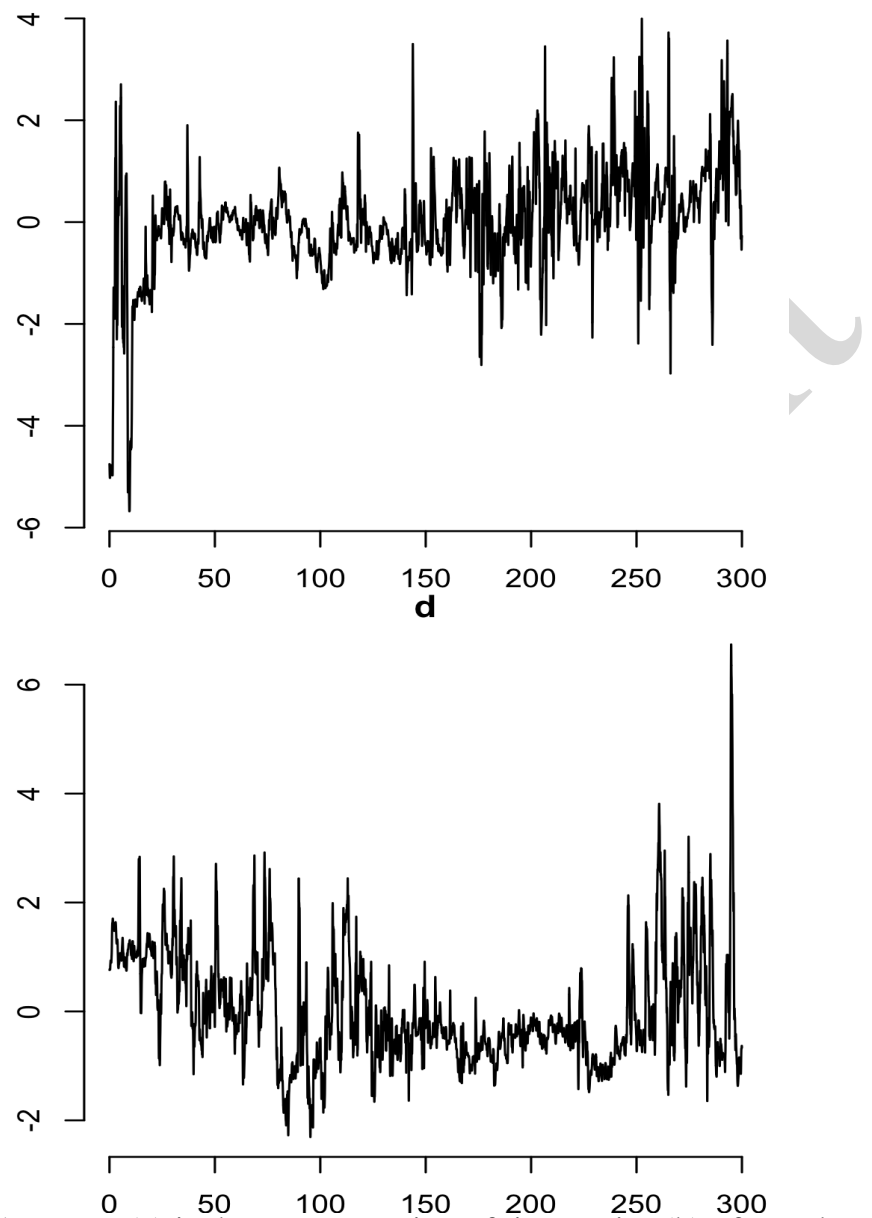

Figure 8. A HAVOK analysis of dyadic head motion data. Attractor (a) is the reconstruction of time series (b) of angular velocities measured of head motion from participant 1. Attractor (c) is the reconstruction of time series (d) of angular velocities measured of head motion from participant 1.

the model implied time series compared to both the original time series and the first eigen time series of $\mathbf{V}$, all while (c) studying the behavior of $\mathbf{u}(\mathbf{t})$ and attractor reconstructed by HAVOK analysis to be sure $\mathbf{u}(\mathbf{t})$ is showing regions of both active and inactive forcing. Given proper selection of these hyper-parameters, HAVOK analysis shows promise of being a near universal modeling framework for nonlinear time series data derived from psychological and behavioral studies.

\section{Limitations}

Although we believe HAVOK analysis to show great potential as a general use analytical method for modeling nonlinear dynamics, there are a number of limitations of HAVOK analysis that may limit the applicability of HAVOK analysis for understanding psychological and behavioral systems. Most of these limitations are due to the relatively new nature of HAVOK analysis and the complexity of human data. Specifically, we do not yet know the minimum num- ber of samples, minimum sampling rate, or relevant signalto-noise ratio required of time series to successfully recover nonlinear dynamics through HAVOK analysis. These numbers are theoretically a function of the dimensionality of the attractor of a system, but this dimensionality may be constantly changing in human systems. Additionally, as it stands HAVOK analysis does not yet have many well-defined statistical attributes. That is, currently within the HAVOK analysis framework there do not yet exist metrics such as fit statistics, $p$-values, or measures of uncertainty such as standard errors. Although in our examples we choose to define fit through the use of $R^{2}$ values, this may not be the optimal measure of fit to use when comparing the results of the HAVOK model to the original time series.

Though the examples we give in this article are a diverse array of time series that a psychological/behavioral researcher might encounter, our examples are by no means exhaustive of the myriad of data streams a researcher may en- 

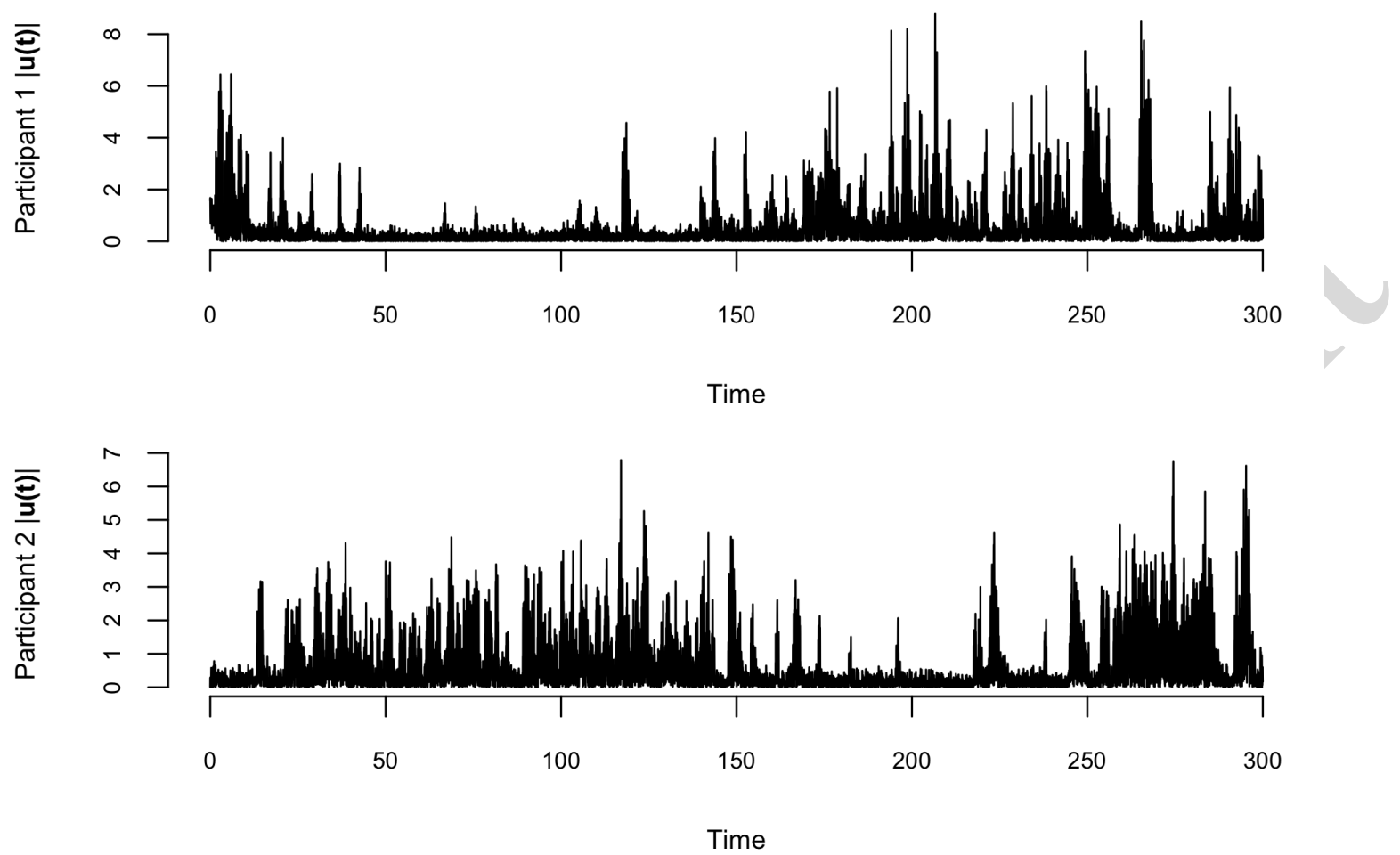

Figure 9. Forcing terms obtained form separate HAVOK analyses of head motions obtained from two participants engaged in dyadic conversation with one another. When the forcing term of one participant becomes active, the forcing term of the other participant becomes inactive.

counter. Other data sets may show differing degrees of nonlinearity, noise, or missingness. Indeed, methods for dealing with missing values are of specific interest to applied researchers since missing data are to be expected in the majority of studies involving data collection over time. Previous research has reported time-delay embedding matrices derived from Takens' embedding theorem to be robust to missing data and sampling misspecification and this may hold for $\mathbf{H}$ as well (Boker, Tiberio, \& Moulder, 2018). Attractors from human system may also change form and structure dependant on other factors like environment and specific situation. HAVOK analysis still needs to be validated on such cases. Another limitation of HAVOK analysis is that there is currently no optimization procedure for best selecting either $r$ or the number of rows in $\mathbf{H}$ as there is not yet a well-defined measure of fit for HAVOK models, though many values of these parameters may show equally good fit (Brunton et al., 2017). Finally, the current implementation of HAVOK analysis also only applicable to univariate time series.

\section{Future Directions}

Perhaps the most exciting aspects of the application of HAVOK analysis to psychological and behavioral time series are the possible future extensions of HAVOK analysis to be better fit to the varied landscape of psychological and behavioral time series. Each limitation of HAVOK analysis appears to be an avenue of research to this end. There currently exist some possible metrics for quantifying the fit of HAVOK models to data sets (e.g., Hamilton, 1986; Kantas, Doucet, Singh, Maciejowski, \& Chopin, 2015). In fact, given that the core of HAVOK analysis is a regression/state-space model it is highly probable that the current 2-step procedure for estimating a HAVOK model (i.e., dimension reduction and then model construction) may be fit simultaneously in a singular structural equation model (SEM) or Gaussian graphical model (GGM) through currently available software (Chow et al., 2010; Epskamp et al., 2018; Neale et al., 2016). This would greatly increase the generality of HAVOK analysis as all of the known forms of SEM and GGM (e.g., multivariate data, mixture-modeling, clustering, categorical data analysis, and robustness to measurement error) would then be applicable to HAVOK. Additional improvements to the HAVOK analysis framework might augmenting equation 10 with additional outside of the univariate time series under study.

Even without these improvements, HAVOK analysis offers psychological and behavioral researchers a new tool for studying nonlinear dynamics in time series data. Areas of research which may be applicable to HAVOK analysis in 

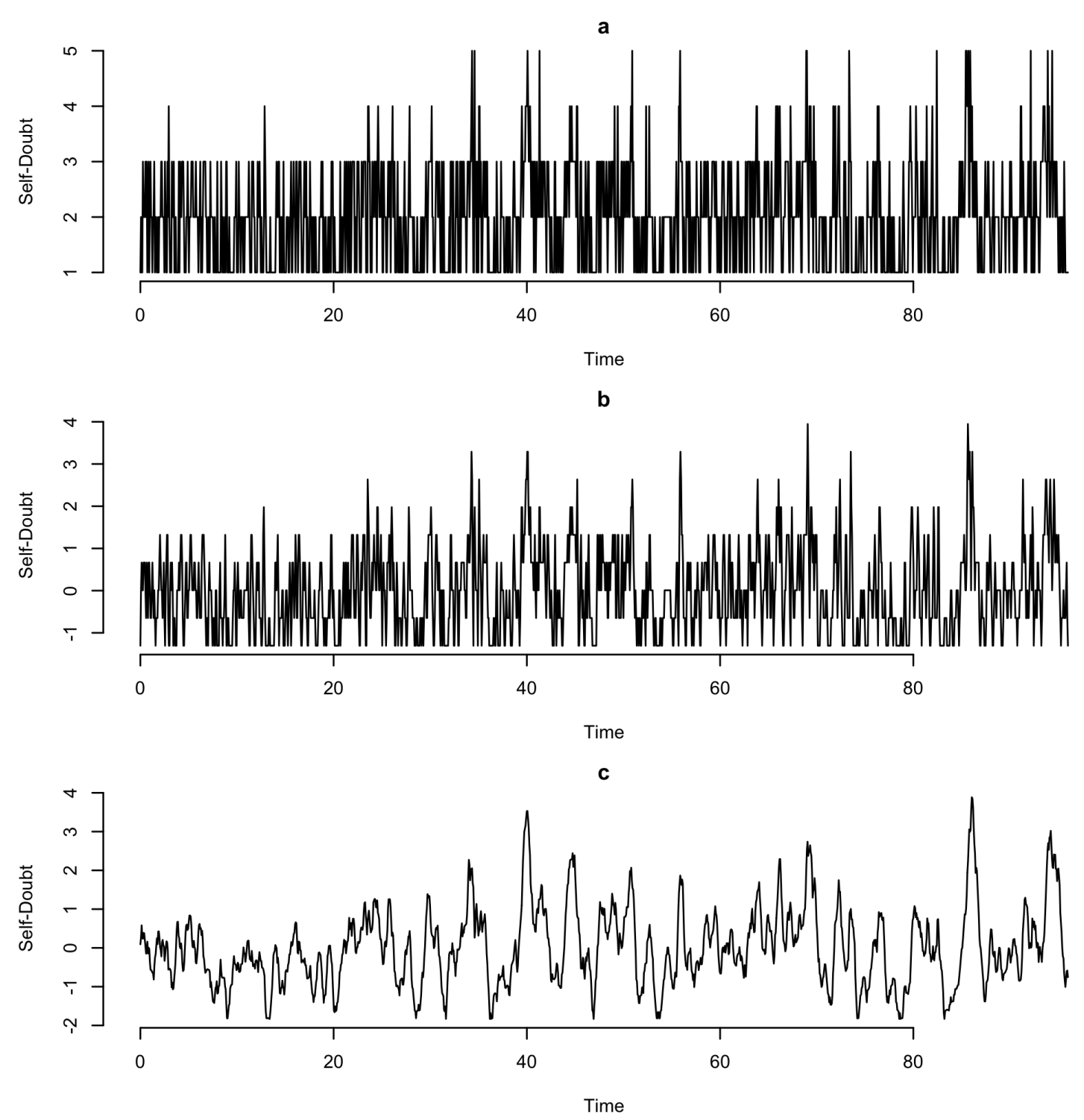

Figure 10. Time series representing (a) raw values of self-reported self-doubt values on a scale of 1 to 7 across 8 months, (b) the first eigen time series of a HAVOK analysis of (a) with an $r$ of 2 and an $\mathbf{H}$ with 2 rows and 1471 columns, and (c) the first eigen time series of a HAVOK analysis of (a) with an $r$ of 2 and an $\mathbf{H}$ with 10 rows and 1463 columns.

its current state are EMA studies and psychophysiological studies, however any study collecting time series data from humans may be applicable to HAVOK analysis. Questions of statistical power and fit are natural next steps for further establishing HAVOK analysis as a useful tool for physiological and behavioral researchers. Most interestingly, HAVOK analysis utilizes a forcing term to perturb a linear system into acting as a nonlinear system. Given that humans act as complex nonlinear dynamical systems, this implies that well-timed perturbations may induce meaningful changes in human behavior and may be useful for just-in-time intervention studies (Nahum-Shani et al., 2018; Shinbrot, Grebogi,
Yorke, \& Ott, 1993; Spruijt-Metz \& Nilsen, 2014).

\section{Conclusion}

We have presented HAVOK analysis as a modeling paradigm that offers researchers a linear framework for modeling nonlinear dynamics. HAVOK analysis can recover meaningful nonlinear dynamics from both theoretical and applied systems of interest to psychological and behavioral researchers. HAVOK analysis also has many possible extensions that could further the usefulness and general appeal of HAVOK analysis for uncovering nonlinear dynamics.

Humans change in many ways. Sometimes these changes 

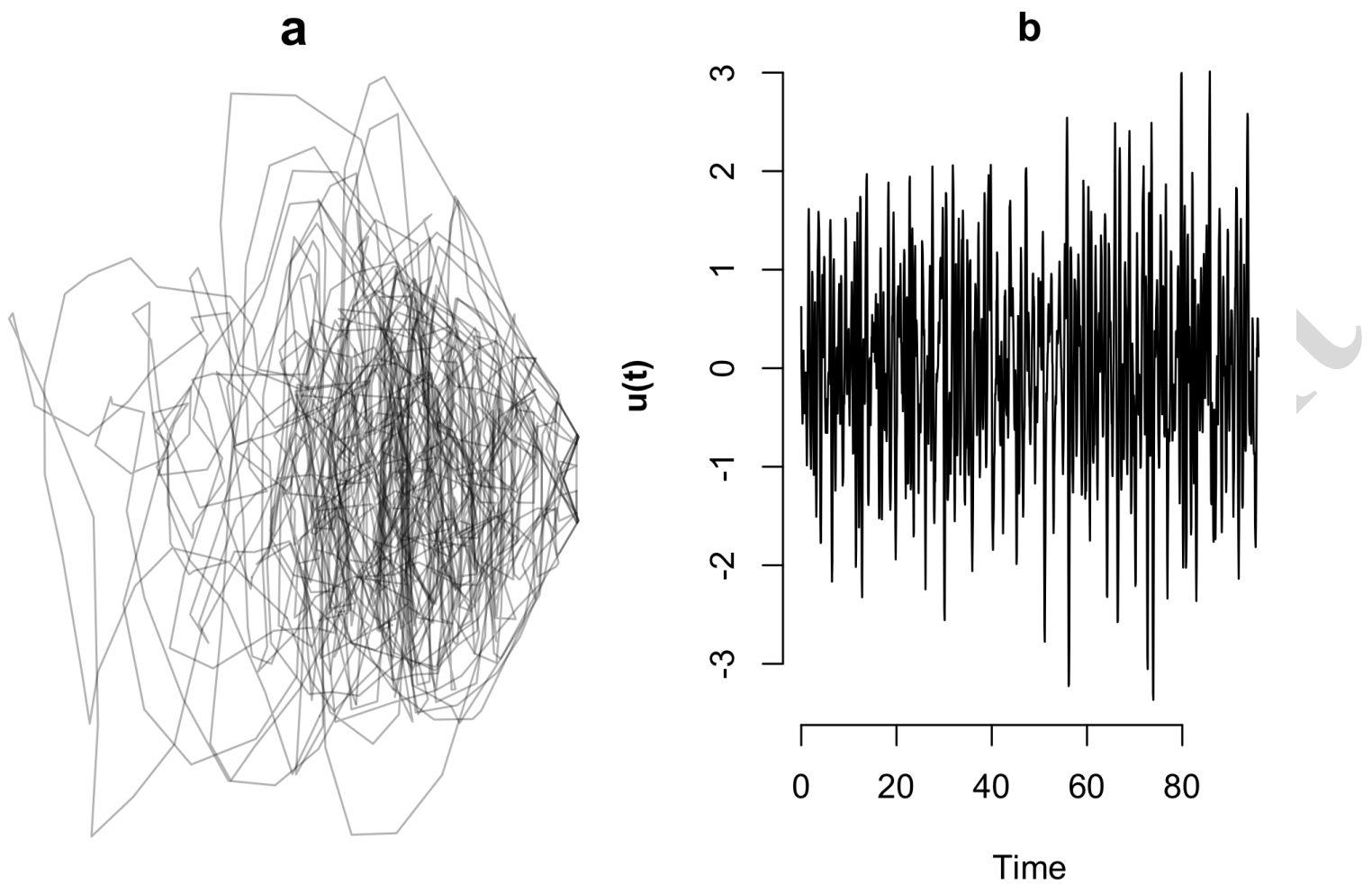

Figure 11. HAVOK analysis of self-reported self-doubt values over time. The attractor of this system (a) shows relatively little structure, but does seem to have a region in which the trajectories are more dense than others. The forcing term of this system (b) is highly erratic, indicating that this HAVOK parameterization of this system is highly nonlinear.

are abrupt, unexpected, and nonlinear. Other times these changes are much more gradual and predictable. Either way, this change may be understood to be a complex and dynamic process. The only way then to understand human experience is to understand and model change as a complex, dynamic, and possibly nonlinear process. As researchers begin to become more interested in studying how individuals change over time, methods such as HAVOK analysis will be necessary as a tool for researchers to explore, test, and generate new hypotheses regarding the way humans change on both short and long time scales. Identifying and targeting nonlinear segments of human experience, as given through the use of HAVOK analysis, may lead to further developments on defining what nonlinearity means for humans, as well as why so much of human behavior is not simple and linear, but complex and adaptive.

\section{References}

Abraham, F. D. (1997). Nonlinear Coherence in Multivariate Research: Invariants and the Reconstruction of Attractors. Nonlinear Dynamics, Psychology, and Life Sciences, 1(1), 7-33. doi: 10.1023/A:1022319825961

Asbury, K., Dunn, J. F., Pike, A., \& Plomin, R. (2003). Nonshared Environmental Influences on Individual Differences in Early Behavioral Development: A Monozygotic Twin
Differences Study. Child Development, 74(3), 933-943. doi: 10.1111/1467-8624.00577

Ashenfelter, K. T., Boker M., S., Waddell, J. R., \& Vitanov, N. (2009). Spatiotemporal symmetry and multifractal structure of head movements during dyadic conversation. Journal of experimental psychology. Human perception and performance, 35(4), 1072-1091. doi: 10.1037/a0015017

Barton, S. (1994). Chaos, self-organization, and psychology. American Psychologist, 49(1), 5-14. doi: 10.1037/0003066X.49.1.5

Behrens, F., Snijdewint, J., Moulder, R. G., Prochazkova, E., SjakShie, E. E., Boker, S. M., \& Kret, M. E. (2019). Physiological synchrony promotes cooperative success in real-life interactions. bioRxiv, 792416. doi: 10.1101/792416

Bhattacharya, J., \& Kanjilal, P. (1999). On the detection of determinism in a time series. Physica D: Nonlinear Phenomena, 132(1-2), 100-110. doi: 10.1016/S0167-2789(99)00033-0

Boker, S. M. (2013). Selection, optimization, compensation, and equilibrium dynamics. GeroPsych. doi: 10.1024/16629647/a000081

Boker, S. M., Deboeck, P. R., Edler, C., \& Keel, P. K. (2010). Generalized local linear approximation of derivatives from time series. In Statistical methods for modeling human dynamics: An interdisciplinary dialogue. (pp. 161-178). New York, NY, US: Routledge/Taylor \& Francis Group.

Boker, S. M., Neale, M., \& Rausch, J. (2004). Latent differential equation modeling with multivariate multi-occasion indica- 
tors. In Recent developments on structural equation models (pp. 151-174). Springer. doi: 10.1007/978-1-4020-19586_9

Boker, S. M., \& Rotondo, J. L. (2002). Symmetry building and symmetry breaking in synchronized movement. Mirror neurons and the evolution of brain and language., 163-171. doi: 10.1016/S0375-9474(01)00406-7

Boker, S. M., Tiberio, S. S., \& Moulder, R. G. (2018). Robustness of time delay embedding to sampling interval misspecification. In Continuous time modeling in the behavioral and related sciences (pp. 239-258). Springer. doi: 10.1007/9783-319-77219-6_10

Bollt, E. M., Li, Q., Dietrich, F., \& Kevrekidis, I. (2018). On Matching, and Even Rectifying, Dynamical Systems through Koopman Operator Eigenfunctions. SIAM Journal on Applied Dynamical Systems, 17(2), 1925-1960. doi: 10.1137/17M116207X

Brick, T. R., Gray, A. L., \& Staples, A. D. (2018). Recurrence quantification for the analysis of coupled processes in aging. The Journals of Gerontology: Series B, 73(1), 134-147. doi: 10.1093/geronb/gbx018

Broomhead, D., \& King, G. P. (1986). Extracting qualitative dynamics from experimental data. Physica D: Nonlinear Phenomena, 20(2-3), 217-236. doi: 10.1016/01672789(86)90031-X

Brunton, S. L., Brunton, B. W., Proctor, J. L., Kaiser, E., \& Kutz, J. N. (2017). Chaos as an intermittently forced linear system. Nature Communications, 8(1), 19. doi: 10.1038/s41467017-00030-8

Brunton, S. L., Brunton, B. W., Proctor, J. L., \& Kutz, J. N. (2016). Koopman Invariant Subspaces and Finite Linear Representations of Nonlinear Dynamical Systems for Control. PLOS ONE, 11(2), e0150171. doi: 10.1371/journal.pone.0150171

Budišić, M., Mohr, R., \& Mezić, I. (2012). Applied Koopmanism. Chaos: An Interdisciplinary Journal of Nonlinear Science, 22(4), 047510. doi: 10.1063/1.4772195

Chow, S.-M., Ho, M.-h. R., Hamaker, E. L., \& Dolan, C. V. (2010). Equivalence and Differences Between Structural Equation Modeling and State-Space Modeling Techniques. Structural Equation Modeling: A Multidisciplinary Journal, 17(2), 303-332. doi: 10.1080/10705511003661553

Chow, S.-M., Witkiewitz, K., Grasman, R., \& Maisto, S. A. (2015). The cusp catastrophe model as cross-sectional and longitudinal mixture structural equation models. Psychological Methods, 20(1), 142-164. doi: 10.1037/a0038962

Daniel, K. E., Daros, A. R., Beltzer, M. L., Boukhechba, M., Barnes, L. E., \& Teachman, B. A. (2020). How Anxious are You Right Now? Using Ecological Momentary Assessment to Evaluate the Effects of Cognitive Bias Modification for Social Threat Interpretations. Cognitive Therapy and Research, 44(3), 538-556. doi: 10.1007/s10608-020-10088-2

Danvers, A. F., Wundrack, R., \& Mehl, M. (2020). Equilibria in personality states: A conceptual primer for dynamics in personality states. European Journal of Personality. doi: 10.1002/per.2239

Day, S., Junge, O., \& Mischaikow, K. (2004). A rigorous numerical method for the global analysis of infinite-dimensional discrete dynamical systems. SIAM Journal on Applied Dy- namical Systems, 3(2), 117-160. doi: 10.1137/030600210

Deboeck, P. R., \& Bergeman, C. S. (2013). The reservoir model: A differential equation model of psychological regulation. Psychological Methods, 18(2), 237-256. Retrieved from http://doi .apa.org/getdoi.cfm?doi=10.1037/a0031603 doi: $10.1037 / \mathrm{a} 0031603$

de Haan-Rietdijk, S., Voelkle, M. C., Keijsers, L., \& Hamaker, E. L. (2017). Discrete-vs. continuous-time modeling of unequally spaced experience sampling method data. Frontiers in psychology, 8, 1849. doi: 10.3389/fpsyg.2017.01849

Delorme, A., Rousselet, G. A., Macé, M. J., \& Fabre-Thorpe, M. (2004). Interaction of top-down and bottomup processing in the fast visual analysis of natural scenes. Cognitive Brain Research, 19(2), 103-113. doi: 10.1016/j.cogbrainres.2003.11.010

Driver, C. C., \& Voelkle, M. C. (2018). Hierarchical bayesian continuous time dynamic modeling. Psychological Methods, 23(4), 774. doi: 10.1037/met0000168

Engert, V., Vogel, S., Efanov, S. I., Duchesne, A., Corbo, V., Ali, N., \& Pruessner, J. C. (2011). Investigation into the crosscorrelation of salivary cortisol and alpha-amylase responses to psychological stress. Psychoneuroendocrinology, 36(9), 1294-1302. doi: 10.1016/j.psyneuen.2011.02.018

Epskamp, S., Waldorp, L. J., Mõttus, R., \& Borsboom, D. (2018). The Gaussian Graphical Model in Cross-Sectional and TimeSeries Data. Multivariate Behavioral Research, 53(4), 453480. doi: 10.1080/00273171.2018.1454823

Feldman, R., Magori-Cohen, R., Galili, G., Singer, M., \& Louzoun, Y. (2011). Mother and infant coordinate heart rhythms through episodes of interaction synchrony. Infant Behavior and Development, 34(4), 569-577. doi: 10.1016/j.infbeh.2011.06.008

Gavish, M., \& Donoho, D. L. (2014). The optimal hard threshold for singular values is $4 / \sqrt{3}$. IEEE Transactions on Information Theory, 60(8), 5040-5053. doi: 10.1109/TIT.2014.2323359

Gruebler, A., \& Suzuki, K. (2014). Design of a Wearable Device for Reading Positive Expressions from Facial EMG Signals. IEEE Transactions on Affective Computing, 5(3), 227-237. doi: 10.1109/TAFFC.2014.2313557

Guastello, S. J., \& Liebovitch, L. S. (2009). Introduction to nonlinear dynamics and complexity. Cambridge University Press.

Hamel, L. M., Moulder, R., Albrecht, T. L., Boker, S. M., Eggly, S., \& Penner, L. A. (2018). Nonverbal synchrony as a behavioural marker of patient and physician race-related attitudes and a predictor of outcomes in oncology interactions: protocol for a secondary analysis of video-recorded cancer treatment discussions. BMJ open, $8(12)$.

Hamilton, J. D. (1986). A standard error for the estimated state vector of a state-space model. Journal of Econometrics, 33(3), 387-397. doi: 10.1016/0304-4076(86)90004-7

Hindmarsh, J. L., \& Rose, R. (1984). A model of neuronal bursting using three coupled first order differential equations. Proceedings of the Royal society of London. Series B. Biological sciences, 221(1222), 87-102. doi: 10.1098/rspb.1984.0024

Hodgkin, A. L., \& Huxley, A. F. (1952). A quantitative description of membrane current and its application to conduction and excitation in nerve. The Journal of physiology, 117(4), 500. 
doi: 10.1113/jphysiol.1952.sp004764

Horn, J. L. (1965). A rationale and test for the number of factors in factor analysis. Psychometrika, 30(2), 179-185. doi: 10.1007/BF02289447

Hsu, C. S. (1980). A Theory of Cell-to-Cell Mapping Dynamical Systems. Journal of Applied Mechanics, 47(4), 931-939. doi: $10.1115 / 1.3153816$

Hu, Y., Boker, S. M., Neale, M., \& Klump, K. L. (2014). Coupled latent differential equation with moderators: Simulation and application. Psychological methods, 19(1), 56. doi: $10.1037 / \mathrm{a} 0032476$

Hunter, M. D. (2018). State Space Modeling in an Open Source, Modular, Structural Equation Modeling Environment. Structural Equation Modeling: A Multidisciplinary Journal, 25(2), 307-324. doi: 10.1080/10705511.2017.1369354

Kan, K.-J., Ploeger, A., Raijmakers, M. E. J., Dolan, C. V., \& Van Der Maas, H. L. J. (2010). Nonlinear epigenetic variance: review and simulations. Developmental Science, 13(1), 1127. doi: 10.1111/j.1467-7687.2009.00858.x

Kantas, N., Doucet, A., Singh, S. S., Maciejowski, J., \& Chopin, N. (2015). On particle methods for parameter estimation in state-space models. Statistical Science, 30(3), 328-351. doi: 10.1214/14-STS511

Koopman, B. O. (1931). Hamiltonian Systems and Transformation in Hilbert Space. Proceedings of the National Academy of Sciences, 17(5), 315-318. doi: 10.1073/pnas.17.5.315

Kossakowski, J. J., Groot, P. C., Haslbeck, J. M. B., Borsboom, D., \& Wichers, M. (2017). Data from 'Critical Slowing Down as a Personalized Early Warning Signal for Depression'. Journal of Open Psychology Data, 5, 1-3. doi: 10.5334/jopd.29

Kunhimangalam, R., Joseph, P., \& Sujith, O. (2008). Nonlinear analysis of EEG signals: Surrogate data analysis. IRBM, 29(4), 239-244. doi: 10.1016/j.rbmret.2007.09.006

Lefèvre, T., Lepresle, A., \& Chariot, P. (2014). An alternative to current psychiatric classifications: a psychological landscape hypothesis based on an integrative, dynamical and multidimensional approach. Philosophy, Ethics, and $\mathrm{Hu}$ manities in Medicine, 9(1), 12. doi: 10.1186/1747-53419-12

Liu, C., Liu, T., Liu, L., \& Liu, K. (2004). A new chaotic attractor. Chaos, Solitons \& Fractals, 22(5), 1031-1038. doi 10.1016/j.chaos.2004.02.060

Lorenz, E. N. (1963). Deterministic Nonperiodic Flow. Journal of the Atmospheric Sciences, 20(2), 130-141. doi: 10.1175/1520-0469(1963)020<0130:DNF>2.0.CO;2

Lu, Z., Hunt, B. R., \& Ott, E. (2018). Attractor reconstruction by machine learning. Chaos: An Interdisciplinary Journal of Nonlinear Science, 28(6), 061104. doi: 10.1063/1.5039508

Mattsson, K., \& Nordström, J. (2004). Summation by parts operators for finite difference approximations of second derivatives. Journal of Computational Physics, 199(2), 503-540. doi: 10.1016/j.jcp.2004.03.001

Mezić, I., \& Banaszuk, A. (2004). Comparison of systems with complex behavior. Physica D: Nonlinear Phenomena, 197(1-2), 101-133. doi: 10.1016/j.physd.2004.06.015

Nagarajan, R. (2005). Local analysis of dissipative dynamical systems. International Journal of Bifurcation and Chaos, 15(05), 1515-1547. doi: 10.1142/S0218127405012971
Nahum-Shani, I., Smith, S. N., Spring, B. J., Collins, L. M., Witkiewitz, K., Tewari, A., \& Murphy, S. A. (2018). Justin-time adaptive interventions (jitais) in mobile health: key components and design principles for ongoing health behavior support. Annals of Behavioral Medicine, 52(6), 446-462. doi: 10.1007/s12160-016-9830-8

Neale, M. C., Hunter, M. D., Pritikin, J. N., Zahery, M., Brick, T. R., Kirkpatrick, R. M., ... Boker, S. M. (2016). Openmx 2.0: Extended structural equation and statistical modeling. Psychometrika, 81(2), 535-549. doi: 10.1007/s11336-0149435-8

Nelson, A., Grahe, J., Ramseyer, F., \& Serier, K. (2014). Psychological Data from an Exploration of the Rapport / Synchrony Interplay Using Motion Energy Analysis. Journal of Open Psychology Data, 2(1), e5. doi: 10.5334/jopd.ae

Peppoloni, L., Lawrence, E. L., Ruffaldi, E., \& Valero-Cuevas, F. J. (2017). Characterization of the disruption of neural control strategies for dynamic fingertip forces from attractor reconstruction. PLOS ONE, 12(2), 1-23. doi: 10.1371/journal.pone. 0172025

Ramseyer, F., \& Tschacher, W. (2011). Nonverbal synchrony in psychotherapy: coordinated body movement reflects relationship quality and outcome. Journal of consulting and clinical psychology, 79(3), 284-295. doi: 10.1037/a0023419

Richardson, M. J., Dale, R., \& Marsh, K. L. (2014). Complex Dynamical Systems in Social and Personality Psychology. In H. T. Reis \& C. M. Judd (Eds.), Handbook of research methods in social and personality psychology (pp. 253-282). New York: Cambridge University Press. doi: 10.1017/CBO9780511996481.015

Santangelo, P., Bohus, M., \& Ebner-Priemer, U. W. (2014). Ecological momentary assessment in borderline personality disorder: a review of recent findings and methodological challenges. Journal of personality disorders, 28(4), 555-576. doi: 10.1521/pedi_2012_26_067

Shinbrot, T., Grebogi, C., Yorke, J. A., \& Ott, E. (1993). Using small perturbations to control chaos. Nature, 363(6428), 411-417. doi: 10.1038/363411a0

Spruijt-Metz, D., \& Nilsen, W. (2014). Dynamic models of behavior for just-in-time adaptive interventions. IEEE Pervasive Computing, 13(3), 13-17. doi: 10.1109/MPRV.2014.46

Stam, C. (2005). Nonlinear dynamical analysis of EEG and MEG: Review of an emerging field. Clinical Neurophysiology, 116(10), 2266-2301. doi: 10.1016/j.clinph.2005.06.011

Steele, J. S., \& Ferrer, E. (2011). Latent differential equation modeling of self-regulatory and coregulatory affective processes. Multivariate Behavioral Research, 46(6), 956-984. doi: 10.1080/00273171.2011.625305

Takens, F. (1981). Detecting strange attractors in turbulence. In Lecture notes in mathematics (Vol. 898, pp. 366-381). doi: 10.1007/BFb0091924

Timofejeva, I., Poskuviene, K., Cao, M., \& Ragulskis, M. (2018). Synchronization measure based on a geometric approach to attractor embedding using finite observation windows. Complexity, 2018. doi: 10.1155/2018/8259496

Turkheimer, E., \& Waldron, M. (2000). Nonshared environment: A theoretical, methodological, and quantitative review. Psychological Bulletin, 126(1), 78-108. doi: 10.1037/0033- 
2909.126.1.78

Vallacher, R. R., \& Nowak, A. (1997). The Emergence of Dynamical Social Psychology. Psychological Inquiry, 8(2), 73-99. doi: 10.1207/s15327965pli0802_1

van Montfort, K., Oud, J. H., \& Voelkle, M. C. (2018). Continuous time modeling in the behavioral and related sciences. Springer.

Wall, M. E., Rechtsteiner, A., \& Rocha, L. M. (2003). Singular value decomposition and principal component analysis. In A practical approach to microarray data analysis (pp. 91109). Springer. doi: 10.1007/0-306-47815-3_5

Weiss, A. A. (1984). Systematic sampling and temporal aggregation in time series models. Journal of Econometrics, 26(3),
271-281. doi: 10.1016/0304-4076(84)90022-8

Whitney, H. (1936). Differentiable Manifolds. The Annals of Mathematics, 37(3), 645. doi: 10.2307/1968482

Wichers, M., \& Groot, P. C. (2016). Critical Slowing Down as a Personalized Early Warning Signal for Depression. Psychotherapy and Psychosomatics, 85(2), 114-116. doi: 10.1159/000441458

Wild, B., Eichler, M., Friederich, H.-C., Hartmann, M., Zipfel, S., \& Herzog, W. (2010). A graphical vector autoregressive modelling approach to the analysis of electronic diary data. BMC Medical Research Methodology, 10(1), 28. doi: 10.1186/1471-2288-10-28 